\title{
Effelsberg multifrequency pulsar polarimetry
}

\author{
A. von Hoensbroech ${ }^{1}$ and K.M. Xilouris ${ }^{2}$ \\ 1 Max-Planck-Institut für Radioastronomie, Auf dem Hügel 69, D-53121 Bonn, Germany \\ 2 Cornell University, National Astronomy \& Ionospheric Center, Arecibo Observatory, P.O. Box 995, Arecibo, PR 00613, U.S.A.
}

Received October 14, 1996; accepted February 24, 1997

\begin{abstract}
A sample of 64 well-calibrated pulsar polarization profiles measured at cm-wavelengths with the Effelsberg radio telescope is presented. All profiles were measured using an adding and a multiplying polarimeter which enables polarimetry over wide-bandwidths. Gain imbalances introduced by the active components in the signal path, as well as system imperfections, alter the polarization state of the incoming radiation. An analysis of the signal path of those devices is presented, together with a dynamic calibration procedure introduced to eliminate the instrumental errors. The results were used to trace the location of the radiating region in pulsar magnetospheres at high frequencies.
\end{abstract}

Key words: polarization — techniques: polarimetric pulsars: general - pulsars: individual PSR B0136+57, PSR B0301+19, PSR B0329+54, PSR B0355+54, PSR B0450+55, PSR B0525+21, PSR B0540+23, PSR B0740-28, PSR B0809+74, PSR B0823+26, PSR B0919+06, PSR B0950+08, PSR B1133+16, PSR B1237+35, PSR B1642-03, PSR B1822-09, PSR B1915+13, PSR B1929+10, PSR B1946+35, PSR B2016+28, PSR B2020+28, PSR B2021+51, PSR B2045-16, PSR B2154+40, PSR B2310+42, PSR B2319+60, PSR B2351+61

\section{Introduction}

Polarimetric studies of pulsar radio emission have contributed significantly to our understanding of both the

Send offprint requests to: A. von Hoensbroech (avh@mpifrbonn.mpg.de) geometry involved, and the emission properties of the radiation generated in the magnetospheres of these stars. A promising technique involving high-quality polarimetric measurements has been put forward by Blaskiewicz et al. 1991 (1991, hereafter BCW), and followed up by (von Hoensbroech \& Xilouris 1997). The classical rotating vector model as developed by Radhakrishnan \& Cooke (1969) was modified by BCW to include first-order relativistic corrections. They predict a time-lag between the centroid of the pulsar-profile and the steepest point of its polarization position angle (PPA) curve. This time lag is proportional to the emission altitude. While the BCW method has been applied successfully to decimeter wavelengths, presumably associated with high-altitude emission regions in the context of the radius-to-frequency mapping model (Cordes 1978), very little is known about emission heights at $\mathrm{cm}$-wavelengths, which correspond to regions much closer to the pulsar surface. Such an investigation is initiated by our observations.

In the present paper, a procedure is presented that dynamically accounts for the differential gain variations introduced by the observing system. In addition, corrections are applied for spurious polarization introduced to the measurements by the system characteristics. The calibrated data are presented here while the procedure to derive emission altitudes from this data set as well as the significance of a stratification of the lower magnetospheric regions in pulsar magnetospheres is discussed in an accompanying paper (von Hoensbroech \& Xilouris 1997).

\section{System description}

Pulsar polarimetry has been performed for the strongest pulsars in the declination range of the Effelsberg 100-m radio telescope using receivers centered at 1.4, 1.7, 4.85 and $10.45 \mathrm{GHz}$. The dispersive properties of the interstellar medium (ISM) tend to degrade the milliperiod resolution attainable with the pulsar data acquisition 
system at Effelsberg, PUB86 (Kramer 1995). Typically, between $1.4-1.7 \mathrm{GHz}$, dispersion smearing is significant for most of the pulsars in our sample, and therefore, a device was employed to compensate for this effect. As dispersion smearing scales inversely with the square of the frequency, observations at frequencies higher than $1.7 \mathrm{GHz}$ were usually performed without the use of a dedispersing device. Dedispersion is handled by an on-line signal processor called the Pulsar Signal Entzerrer, (PSE). This device is basically a four unit, 60 channel $\times 667-\mathrm{kHz}$ filterbank providing $40-\mathrm{MHz}$ total bandwidth in each unit. The output of each channel is detected and converted to a digital signal by a fast A/D converter. After a time delay selectable by the user to counteract the effect of dispersion smearing, the outputs of all channels are summed, and the total $40-\mathrm{MHz}$ bandwidth is then recorded by the PUB86. To provide both high time resolution polarimetry and profiles with high signal-to-noise ratio (snr), wide bandwidths were needed. Therefore, two different types of polarimeters were employed. When dispersion smearing significantly degraded the resolution of our profiles, an adding polarimeter was used. This device consists of a network of passive hybrids that produce a maximum bandwidth of $40 \mathrm{MHz}$. Where dispersion was not significant, a multiplying polarimeter was used to handle bandwidths of up to $500 \mathrm{MHz}$. This is a correlation device which derives the linear component of the incident radiation by correlating two nominally circular inputs.

Differential complex gains introduced by various components in the signal path, as well as system imperfections, alter the polarization state of the incoming radiation. We present here a full gain analysis of both polarimeters and describe the procedure followed to monitor gain variations and then account for them during the off-line reduction. We will also refer to the cross-coupling correction; an effect which tends to be stable with time. However, gain variations demand dynamic monitoring if high-precision polarimetry is required.

\section{Polarimeters and the calibration}

The polarization state of a wave incident on a polarizer in front of the first amplification stage of a receiver can be described by two circular components. Following Kraus (1966), these components can be expressed as:

$$
\begin{aligned}
& \mathrm{RHC} \equiv R=r \cdot \mathrm{e}^{j \omega t}=r \cdot \mathrm{e}^{j \psi_{r}} \\
& \mathrm{LHC} \equiv L=l \cdot \mathrm{e}^{-j(\omega t+\phi)}=l \cdot \mathrm{e}^{j \psi_{l}},
\end{aligned}
$$

where $r, l$ represent the electric vector amplitude of each of the circularly polarized components, and $\delta=\psi_{r}-\psi_{l}$ their relative phase. In reality, the incoming signal is elliptically polarized due to the properties of the source, imperfections in the reflector, the feeds, and the polarizer. Measurements of continuum sources (Turlo et al. 1985) or pulsars (Stinebring et al. 1984; Xilouris 1991) can estimate those imperfections introduced by the receiving system which tend to be stable over long time periods. To derive full polarization information, a correlation device is often employed which produces power outputs proportional to the Stokes parameters defined as follows (see Saikia \& Salter 1988):

$$
\begin{aligned}
I & =\left\langle R^{2}+L^{2}>\right. \\
V & =<L^{2}-R^{2}> \\
Q & =<L^{*} R+L R^{*}>=2<r l \cos \delta> \\
U & =\left\langle L^{*} R-L R^{*}>=2<r l \sin \delta>,\right.
\end{aligned}
$$

where $\langle\ldots\rangle$ denotes time averaging. The Stokes parameters are used to characterize the polarization state of the incident radiation. The parameter $I$ represents the total power, $V$ the circularly polarized power, while the linearly polarized power $(L)$ is the vector sum of Stokes parameters $Q$ and $U$. The PPA is given as $\psi=\frac{\delta}{2}=\frac{1}{2} \arctan \frac{U}{Q}$. The polarization state of the incident radiation is degraded further by phase and gain imbalances introduced by the various amplification stages between the two channels following the polarizer as well as by the correlation devices used to produce the Stokes parameters. The gain imbalances are time dependent, and for high quality polarimetry, they must be continuously monitored and dynamically corrected for. In the following sections, our approach to the problem of dynamic calibration is presented for both the adding and multiplying polarimeters which we used.

\subsection{Description of an adding polarimeter}

The adding polarimeter is a passive device which performs analog phase-correct additions of the two incoming signals before their detection. This type of polarimeter is used when further analog signal processing (i.e. via a filter bank) follows.

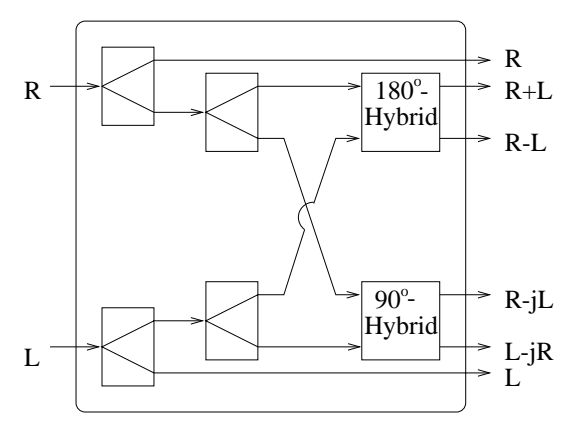

Fig. 1. Block diagram of the $40-\mathrm{MHz}$ adding polarimeter at Effelsberg. The RCH and LHC signals are split into three pairs. Each two pairs, appropriately delayed in phase are added by the quadrature and $180^{\circ}$ hybrids, resulting in the denoted signal outputs 
The adding polarimeter consists of a network of $90^{\circ}$ and $180^{\circ}$ hybrids which perform phase-correct additions with wide-band response and minimal loss. It is this quality of wide-band analog response that has made them the best approach for high-frequency wide-bandwidth polarimetry. Generally, an adding polarimeter can provide six analog outputs (see Fig. 1). The data acquisition system at Effelsberg PUB86, is capable of synchronously detecting only 4 inputs. We chose to record the LHC and RHC components separately and used these values to furnish the total intensity because it is straightforward to convert the measured deflections into flux densities. To make an absolute flux-density calibration, one compares the noise-source deflections with those of standard continuum sources. The voltages provided by each output of the adding polarimeter are shown in Fig. 1. Following squarelaw detection, the power detected in each of the four chosen outputs is given by:

Channel $1=<|R|^{2}>=<R \cdot R^{*}>$

Channel $2=<|L|^{2}>=<L \cdot L^{*}>$

Channel $\left.3=<|R-L|^{2}\right\rangle=<(R-L) \cdot(R-L)^{*}>$

Channel $4=<|L-j R|^{2}>=<(L-j R) \cdot(L-j R)^{*}>$,

where the $*$ denotes the complex conjugate of a vector. The Stokes parameters can then be derived as:

$$
\begin{aligned}
I & =\text { Channel } 1+\text { Channel } 2 \\
V & =\text { Channel } 2-\text { Channel } 1
\end{aligned}
$$

$$
\begin{aligned}
\text { Channel } 3 & =<(R-L)(R-L)^{*}> \\
& =<R R^{*}+L L^{*}-R L^{*}-L R^{*}> \\
& =I-<r l\left(\mathrm{e}^{j\left(\psi_{r}-\psi_{l}\right)}+\mathrm{e}^{-j\left(\psi_{r}-\psi_{l}\right)}\right)> \\
& =I-<r l\left(\mathrm{e}^{j \delta}+\mathrm{e}^{-j \delta}\right)> \\
& =I-<2 r l \cos \delta> \\
& =I-Q \\
\Rightarrow Q & =I-\text { Channel } 3
\end{aligned}
$$

and

$$
\text { Channel } \begin{aligned}
4 & =<(R-j L)(R-j L)^{*}> \\
& =<R R^{*}+L L^{*}+j R L^{*}-j L R^{*}> \\
& =I-j<r l\left(\mathrm{e}^{j\left(\psi_{r}-\psi_{l}\right)}-\mathrm{e}^{j\left(\psi_{r}-\psi_{l}\right)}\right)> \\
& =I-j<r l\left(\mathrm{e}^{j \delta}-\mathrm{e}^{-j \delta}\right)> \\
& =I+<2 r l \sin \delta> \\
& =I+U \\
\Rightarrow U & =\text { Channel } 4-I .
\end{aligned}
$$

Prior to subtraction, a scaling factor is necessary between the total power $I$ and the outputs of channels 3 and 4 in order to produce the linear components $Q$ and $U$. Such scaling is necessary because of the gain differences between the paths of channels 3 and 4 , and the total power $I$.

\subsubsection{Dynamic calibration}

The quadrature and $180^{\circ}$ hybrids have been measured to introduce with great repeatability their nominal gain and phase delays in the circular components of the propagating signals. Other active components in the propagation path such as amplifiers, and phase shifters, as well as any piece of wave guide, introduce differential gains and phases between the two circular components. These differences are reflected in the Stokes parameters as spurious polarization and therefore they have to be detected and accounted for. To detect and monitor the stability of these gains, a noise signal is fired synchronously to the pulsar period into the receiver horn prior to the polarizer. The injection scheme is such that most of this calibration signal propagates towards the receiver and not the reflector. To get a correlated calibration signal, the power from a single diode is split and fed into the feed horn. A visual conception of the gain factors involved in the system is presented in the following block diagram in Fig. 2. The gains along the prop-

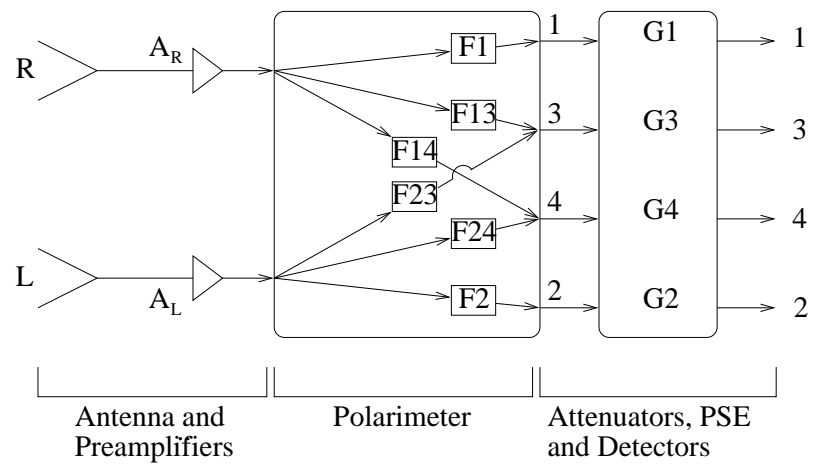

Fig. 2. Different gain-factors of the system

agation path can be identified in three different groups. Following Rankin et al. (1975), the gains introduced in the path between the reflector and the entrance to the polarimeter are symbolized as $A_{R}$ and $A_{L}$. The gains between the inputs and the outputs of the polarimeter are symbolized as $F_{1}, F_{2}, F_{13}, F_{14}, F_{23}$ and $F_{24}$. The gains introduced by the variable attenuators, the dedisperser, and the analog to digital detectors are symbolized as $G_{1}$ to $G_{4}$. The various gains will influence the measured power in the output of each channel as:

Channel $1=A_{R} F_{1} G_{1} \cdot<|R|^{2}>$

Channel $2=A_{L} F_{2} G_{2} \cdot\left\langle|L|^{2}>\right.$

Channel $3=<A_{R} F_{13} \cdot|R|^{2}+A_{L} F_{23} \cdot|L|^{2}-$ 


$$
\begin{aligned}
\text { Channel } 4= & -\sqrt{A_{R} A_{L} F_{13} F_{23}} \cdot Q>\cdot G_{3} \\
& -\sqrt{A_{R} F_{14} \cdot|R|^{2} F_{14} F_{24}} \cdot U>A_{L} F_{24} \cdot|L|^{2}-
\end{aligned}
$$

with $R$ and $L$ as the two circular components which represent real signals. The main variations will occur in the $A$ - and $G$-gains. The polarimeter-gains $(F)$ were found to be relatively constant due to the passive components in the signal path. In particular, the products $F_{13} \cdot F_{23}$ and $F_{14} \cdot F_{24}$ should be equal. If this is the case, then we can set $A_{R} A_{L} F_{13} F_{23}=A_{R} A_{L} F_{14} F_{24} \equiv \alpha^{2}$. Further, we assume that $A_{R} F_{13} G_{3} \cdot R+A_{L} F_{23} G_{3} \cdot L \equiv g_{3} \cdot I$ and $A_{R} F_{14} G_{4} \cdot R+A_{L} F_{24} G_{4} \cdot L \equiv g_{4} \cdot I$. Thus, the system of Eqs. (9) can be simplified as:

$$
\begin{aligned}
& \text { Channel } 1=g_{1} \cdot\left\langle|R|^{2}>\right. \\
& \text { Channel } 2=g_{2} \cdot\left\langle|L|^{2}>\right. \\
& \text { Channel } 3=g_{3} \cdot\langle I-\alpha Q> \\
& \text { Channel } 4=g_{4} \cdot\langle I-\alpha U>.
\end{aligned}
$$

The remaining five gains $\left(g_{1}-g_{4}, \alpha\right)$ can now be calculated when the following assumptions are made for the calibration noise-signal: (a) the noise-signal is $100 \%$ linearly polarized, which requires the intensity in channels 1 and 2 to be equal and (b) the PPA of the signal is set at $0^{\circ}$. Deviations from this would result in unwanted terms inserted in the calculations. The signals in channels 1 and 2 have to be calibrated to the same deflection levels. In channel $3(=I-Q)$, no signal is expected because $Q=I \cos 2 \psi=I$ when $\psi=0^{\circ}$. In channel $4(=I-U)$, we expect the maximum signal because, $U=I \sin 2 \psi=0$ when $\psi=0^{\circ}$. This is accomplished by introducing the necessary delays via a "delay-box". This is a unit that allows extra electrical length in the signal path so that the calibration signal as monitored on an oscilloscope display following the multiplying polarimeter has all the power in the $U$ channel while there is virtually no power in the $Q$ channel. This is checked over the entire bandwidth and the phases are adjusted in such a way as to put as few zero crossings in the $Q$ channel as possible thereby, ensuring that the polarimeter operates within the white-light fringe regime. This calibration is performed before each run. For the two year period of these polarization experiments, the phase delays that we had to introduce were very stable with time. Changes were only encountered when a component in the propagation path was deliberately changed or malfunctioning. With $\mathrm{Cal}_{1}$ to $\mathrm{Cal}_{4}$ representing the power of the calibration-signal in the four channels, we obtain the four gain-factors:

$$
\begin{aligned}
g_{1} & =\mathrm{Cal}_{1} \\
g_{2} & =\mathrm{Cal}_{2} \\
g_{3} & =\frac{\mathrm{Cal}_{4}}{2} \frac{\mathrm{rms}_{3}}{\mathrm{rms}_{4}} \\
g_{4} & =\frac{\mathrm{Cal}_{4}}{2}
\end{aligned}
$$

$$
\alpha=1-\frac{\mathrm{Cal}_{3}}{\mathrm{Cal}_{4}} \frac{\mathrm{rms}_{4}}{\mathrm{rms}_{3}}
$$

where $\alpha$ should be close to unity. The $\mathrm{rms}_{3}$ and $\mathrm{rms}_{4}$ give the size of the statistical noise, measured over a baseline in the off-pulse region of channels 3 and 4 . To obtain $g_{3}$ and $\alpha$, one has to normalize channel 3 to channel 4 . Therefore, channel 3 is scaled to the same rms level as channel 4 . The factor of 2 in channels 3 and 4 is introduced because the total intensity of the noise-signal is measured in channel 4 , whereas only half of the noise signal is measured in channels 1 and 2 . The calibration signal is given in units of half of the noise-signal. The Stokes-Parameters are then calculated as follows:

$$
\begin{aligned}
I & =\frac{1}{g_{1}} \text { Channel } 1+\frac{1}{g_{2}} \text { Channel } 2 \\
V & =\frac{1}{g_{2}} \text { Channel } 2-\frac{1}{g_{1}} \text { Channel } 1 \\
Q & =\frac{1}{\alpha}\left(I-\frac{1}{g_{3}} \text { Channel } 3\right) \\
U & =\frac{1}{\alpha}\left(I-\frac{1}{g_{4}} \text { Channel } 4\right)
\end{aligned}
$$

We dynamically monitor the gain values for each pulsar as the calibration signal is synchronously introduced to the pulsar period at the first 50 bins of each pulsar period. These gains were found to be very stable in their ratios for long observing runs. Furthermore, when different ratios were detected this indicated a malfunction of the system.

\subsection{Multiplying polarimeter}

The multiplying polarimeter is easier to calibrate than the adding polarimeter. A multiplying polarimeter works on the principle of correlating the input signals. If the two circular components carry radiation that is linearly polarized, the signals will be $100 \%$ correlated. In one of the branches, a $90^{\circ}$ phase shift is induced which enables the estimation of all four Stokes parameters. The response of this device is linear for the bandwidths of interest.

\subsubsection{The Stokes-parameters}

The outputs of the multiplying polarimeter are as follows:

Channel $\left.1=<|R|^{2}\right\rangle=R \cdot R^{*}$

Channel $2=<|L|^{2}>=L \cdot L^{*}$

Channel $3=r l \cdot \cos \delta$

Channel $4=r l \cdot \sin \delta$,

and therefore, the Stokes parameters are given by:

$$
\begin{aligned}
I & =\text { Channel } 1+\text { Channel } 2 \\
V & =\text { Channel } 2-\text { Channel } 1 \\
Q & =2 \cdot \text { Channel } 3 \\
U & =2 \cdot \text { Channel } 4 .
\end{aligned}
$$




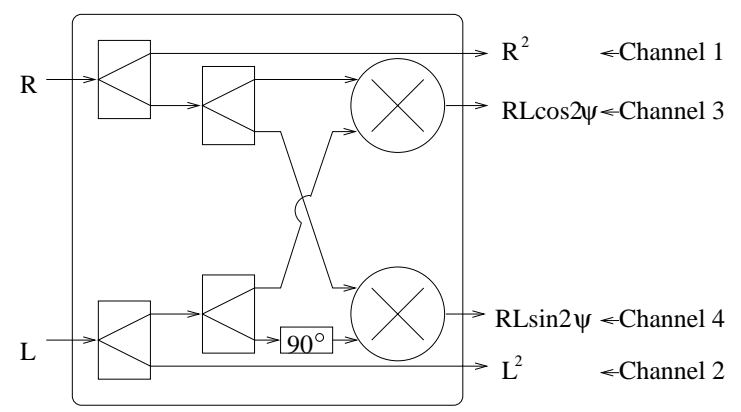

Fig. 3. Block diagram of the multiplying polarimeter. A three-way power splitter feeds the RHC and LHC signals into the input of broadband mixers. In one of the branches, a $90^{\circ}$ phase delay is introduced, to allow for all Stokes parameters to be derived

\subsubsection{Calibration}

It is again helpful to consider the different gain-factors (see Fig. 4) introduced by the observing set-up. The calibration

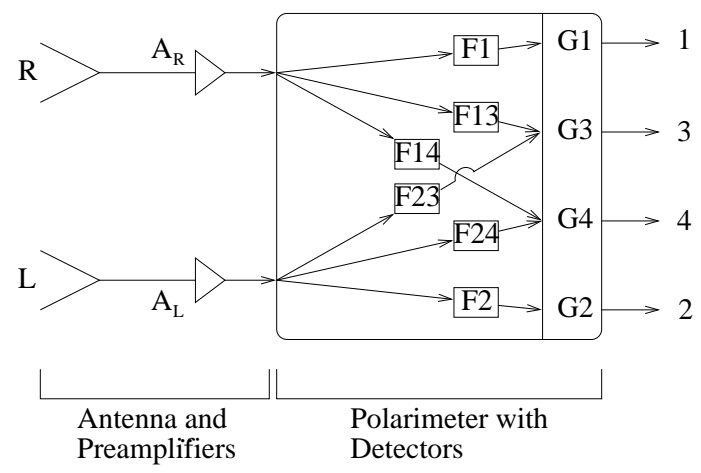

Fig. 4. Different gain-factors of the system

is much easier in this case because the signal is detected directly so there are no vector additions involved (Eq. 15). Using the same definitions for the gains as for the adding polarimeter, the gain propagation along the signal path is described as follows:

$$
\begin{aligned}
& \text { Channel } 1=A_{R} F_{1} G_{1} \cdot\left\langle|R|^{2}>\right. \\
& \text { Channel } 2=A_{L} F_{2} G_{2} \cdot\left\langle|L|^{2}>\right. \\
& \text { Channel } 3=\sqrt{A_{R} A_{L} F_{13} F_{23}} \cdot G_{3} \cdot r l \cos \delta \\
& \text { Channel } 4=\sqrt{A_{R} A_{L} F_{14} F_{24}} \cdot G_{4} \cdot r l \sin \delta .
\end{aligned}
$$

Because there are only multiplicative gains involved, the set of Eq. (15) can be simplified without further assumptions by introducing Eq. (3) as follows:

Channel $1=g_{1} \cdot\left\langle|R|^{2}>\right.$

Channel $2=g_{2} \cdot\left\langle|L|^{2}\right\rangle$
Channel $3=g_{3} \cdot \frac{1}{2} Q$

Channel $4=g_{4} \cdot \frac{1}{2} U$.

To derive the gain-factors, a noise-signal is used in the same way as with the adding polarimeter. However, here we only need to assume that the noise-signal is $100 \%$ linearly polarized. The PPA of the signal can then be calculated from channels 3 and 4 as:

$\psi_{\mathrm{Cal}}=\frac{1}{2} \arctan \left(\frac{\mathrm{Cal}_{4}}{\mathrm{Cal}_{3}} \frac{\mathrm{rms}_{3}}{\mathrm{rms}_{4}}\right)$.

The following gain-factors are then derived:

$g_{1}=\mathrm{Cal}_{1}$

$g_{2}=\mathrm{Cal}_{2}$

$g_{3}=\frac{\mathrm{Cal}_{3}}{\cos 2 \psi_{\mathrm{Cal}}}$

$g_{4}=\frac{\mathrm{Cal}_{4}}{\sin 2 \psi_{\mathrm{Cal}}}$.

Finally, the Stokes parameters can be calculated and normalized to units of half the noise-signal:

$I=\frac{1}{g_{1}}$ Channel $1+\frac{1}{g_{2}}$ Channel 2

$V=\frac{1}{g_{2}}$ Channel $2-\frac{1}{g_{1}}$ Channel 1

$Q=2 \cdot \frac{1}{g_{3}}$ Channel 3

$U=2 \cdot \frac{1}{g_{4}}$ Channel 4 .

The linear $(L)$ and circular $(V)$ polarized intensity and PPA can then be calculated as described in Sect. (3). The error of the PPA was calculated as:

$\Delta \psi=\frac{1}{2\left(Q^{2}+U^{2}\right)} \cdot \sqrt{\left(Q \cdot \mathrm{rms}_{U}\right)^{2}+\left(U \cdot \mathrm{rms}_{Q}\right)^{2}}$.

The error of the polarized linear and circular intensities is estimated by the variance calculated in their off-pulse position of the very nearly Gaussian fluctuations in the Stokes parameters $Q, U$ and $V$ respectively. Typically, each sub-integration lasted for a few hundred pulses. Due to the lengthy integrations and the low gain of the telescope at high frequencies, it is not necessary to correct for bright pulses which could increase the overall system temperature $T_{\text {sys. }}$. The statistics of the linearly polarized intensity are directly derived from the average baseline level of the array containing the linear polarization. Following the instrumental correction procedure, polarization profiles of the same source from different epochs were sometimes added to produce high signal-to-noise profiles that we used for our fitting purposes. To enable this adding, the parallactic angle of each integration was referenced to zero. This transformation was performed on the PPA curve following every instrumental calibration. 
Table 1. The values of the cross-coupling parameters, amplitude $\epsilon$ and phase $\theta$, are shown for all the Effelsberg receivers used in this two year polarization survey

\begin{tabular}{lcll}
\hline $\begin{array}{l}\text { Central } \\
\mathrm{RF}[\mathrm{GHz}]\end{array}$ & $\begin{array}{c}\mathrm{BW} \\
{[\mathrm{MHz}]}\end{array}$ & Ampl. & $\begin{array}{l}\text { Phase } \\
{\left[{ }^{\circ}\right]}\end{array}$ \\
\hline 1.4 & 40 & 0.1 & $68^{\dagger}$ \\
1.4 & 80 & 0.08 & 37 \\
1.7 & 40 & 0.08 & $67^{\dagger}$ \\
4.85 & 500 & 0.019 & 49 \\
10.55 & 300 & 0.08 & 60 \\
\hline
\end{tabular}

${ }^{\dagger}$ observations made with the adding polarimeter.

\subsection{Cross-coupling}

Differential gains, cross-coupling and depolarization will transform the incident polarization state $S(I, Q, U, V)_{\text {true }}$ to the observed $S(I, Q, U, V)_{\text {obs. }}$. The origin of these effects is discussed by Stinebring et al. (1984) and Xilouris (1991), while an evaluation of the instrumental polarization performance of Effelsberg radio telescope at $1.7 \mathrm{GHz}$ was presented by Xilouris (1991). Typically, most of the feeds used have an elliptical rather than a circular response. This means that the axial ratio $\rho$ of the LHC and RHC polarized components of the antenna response is not equal, but very close to, unity. Even if the response is elliptical rather than circular, the feeds can be orthogonal to one another resulting in the antenna fully responding to an arbitrarily polarized wave. Assuming an orthogonally responding antenna, and following proper gain calibration procedures, the cross-coupling will have the net effect of mixing the Stokes parameters. Under the assumption that the amplitude of this effect $\epsilon$ is small $(\sqrt{\epsilon} \ll 1)$, the Stokes parameters are modified to first order as:

$$
\begin{aligned}
I_{\text {obs. }} & =I_{\text {true }} \\
V_{\text {obs. }} & =V_{\text {true }}+L_{\text {true }} \cdot \sqrt{\epsilon} \cos 2(\phi-\theta) \\
Q_{\text {obs. }} & =Q_{\text {true }}-V_{\text {true }} \cdot \sqrt{\epsilon} \cos (2 \theta) \\
U_{\text {obs. }} & =U_{\text {true }}-V_{\text {true }} \cdot \sqrt{\epsilon} \sin (2 \theta)
\end{aligned}
$$

where $\theta$ and $\epsilon$ are the cross-coupling phase and amplitude. Here, $\phi=(\chi+n)$ represents the angle between the effective dipole axis of the antenna and the electric vector of the incident polarization state $S(I, Q, U, V)_{\text {true }}$, while $\chi$ is the true PPA and $n$ the parallactic angle. In most pulsars, the circularly polarized component is small compared to the linear polarization. Assuming $\epsilon$ to be small which is usually the case for most radio telescopes, the effect of cross-coupling on the observed polarization state can be further simplified as:

$$
L_{\text {obs. }}=L_{\text {true }}
$$

$\frac{V_{\text {obs. }}}{L_{\text {obs. }}}=\frac{V_{\text {true }}}{L_{\text {true }}}+\sqrt{\epsilon} \cos 2(\phi-\theta)$.

When measurements of the incident polarization state $S(I, Q, U, V)_{\text {true }}$ are conducted over a sufficient range of parallactic angle $n$, or equivalently, effective parallactic angle $\phi$, the ratio of $\frac{V_{\text {obs }}}{L_{\text {obs }}}$ will exhibit a sinusoidal behavior. The cross coupling parameters $\epsilon$ and $\theta$ are readily obtained from the amplitude and phase of a cosine wave fit to the data. Two well studied and bright pulsars, PSR B1929+10 and PSR B0355+54, were chosen as calibrators and were observed for a small range of parallactic angle during each observing run. Those pulsars apart from exhibiting standard polarization profiles, involve a number of different polarization states with strong linear polarization and small circular, with the associated PPA well defined. This provides a good estimate of the ratio $\frac{V_{\text {obs }}}{L_{\mathrm{obs}}}$ as a function of $\phi$. The fitted parameters $\epsilon$ and $\theta$ were used to correct the $L$ and $V$ waveforms as well as the pulsar intrinsic polarization position angle curve by solving the system of Eqs. (21) for the true polarization states. The results from a two year monitoring of the polarization properties for all of the receivers that we used are presented in Table 1. The cross-coupling amplitude was less than $10 \%$ and quite stable within the time intervals that the instrumental set-up remained unchanged.

A known source of depolarization is Faraday rotation which occurs both in the galactic interstellar medium and in the Earth's ionosphere. These effects combine with unequal phase delays between the two polarization channels of the instrumental setup to effectively depolarize the incident polarization state. In all cases, this effect results from different propagation velocities between the left-hand and right-hand circularly polarized components. The net effect of either true or instrumental Faraday rotation is that the position angle of the linear polarization becomes frequency dependent. Across some finite bandwidth, the dispersion of the position angle results in a net loss of linear polarization. Both the shape and the width of the IF filters that are used to form gain corrected Stokes parameters play an important role in a quantitative determination of the depolarization. Following the analysis of Rankin et al. (1975) for square filters, the largest amounts of depolarization estimated for the highest rotation measure pulsars in our sample at $1.4 \mathrm{GHz}$ is between 3 to $8 \%$ with the rest of the sources less than $1 \%$. At frequencies higher than $1.4 \mathrm{GHz}$, this effect was negligible. The Faraday-induced position angle rotation across the bandwidths used was between $16^{\circ}$ and $32^{\circ}$ for the high RM pulsars in our sample. Most of the RMs in our sample were low and in these cases less than $5^{\circ}$ of rotation were calculated. The instrumentally-induced position angle rotation across the bandwidths used is proportional to the difference in electrical path length between the two circular channels. Severe depolarization would occur if the time delay exceeded the reciprocal of the observing bandwidth. For the $1.4 \mathrm{GHz}$ setup, a difference in the electrical path 
of $7.5 \mathrm{~m}$ would be required to cause severe instrumental depolarization.

\section{Results}

An elaborate procedure was developed to ensure high quality pulsar polarimetry at wide bandwidths. This scheme has served reasonably well for detecting instrumental errors on-line and for monitoring of the polarization characteristics of our instrumental set-up for a period of 2 years at the Effelsberg radio telescope. This procedure was applied to 64 polarization profiles of 28 pulsars at $\mathrm{cm}$-wavelengths. The calibrated polarization profiles are shown in Figs. 5-24 at the end of this section. Whenever possible, a fit of the Rotating-Vector-Model (RVM; e.g. Manchester \& Taylor 1977) to the PPA curve of each source was made which enabled a determination of the viewing geometry of each system. The viewing geometry of each system is presented in Table 3 while the fits are superimposed on the polarization plots of Figs. 5-24. The magnetic inclination angle $\alpha$ and the the impact parameter $\sigma$ (the closest approach of the magnetic axis to the lineof-sight trajectory) are listed in Cols. 3 and 4 of Table 3 respectively, together with their estimated errors. The values of $\alpha$ have a certain ambiguity, because the parameters $\alpha$ and $\sigma$ are highly correlated. This accounts for the large errors associated with these parameters. Thus, it is doubtful whether $\alpha$ always represents the "true" value of the magnetic inclination. However, the BCW method that we have used to determine emission heights at $\mathrm{cm}$-wavelengths is insensitive to the magnetic inclination. Therefore, our results for the emission altitudes are not effected by this ambiguity. The derived heights are presented in the last column of Table 3. A detailed description of the fitting procedure and its associated complications as well as a discussion of our findings is presented in von Hoensbroech \& Xilouris (1997).

In Table 3, quantitative polarization parameters are summarized. The cut-level given in Col. 3 represents the height above the noise (relative to the peak of the respective outer component) where the outer edges of a profile were found. The resulting width of the profile is then listed in the next column. The linear and circular intensities are the relative polarized power integrated over this width. Low polarization levels which become progressively less with frequency are found for most of our sources. Additionally, the flux density was determined and listed in Table 3, together with the integration time of the particular observation.

Polarization plots for all the pulsars in our sample at many frequencies are presented in Figs. 5-24. For each polarization plot, the upper panel contains the total intensity $I$ (solid line), the linearly polarized $L$ (dashed line), and the circularly polarized $V$ (dotted line) intensities. The lower panel contains the derived PPA curve with associated errors at each point. The points were chosen so that
Table 2. The derived geometry and emission heights for each pulsar as a result the fitting procedure. The magnetic inclination, $\alpha$, is the angle between the rotation- and the magnetic axis, while $\sigma$ represents the impact angle between the magnetic axis and the line-of-sight. The emission height derived with the $\mathrm{BCW}$-method is presented on the last column

\begin{tabular}{|c|c|c|c|c|}
\hline PSR B & $\begin{array}{l}\text { Freq. } \\
{[\mathrm{GHz}]}\end{array}$ & $\begin{array}{l}\alpha \\
{\left[{ }^{\circ}\right]}\end{array}$ & $\begin{array}{l}\sigma \\
{\left[{ }^{\circ}\right]}\end{array}$ & $\begin{array}{l}r_{\text {delay }} \\
{[\mathrm{km}]}\end{array}$ \\
\hline $0136+57$ & 4.85 & $96.0 \pm 15.0$ & $-3.6 \pm 15.0$ & $530 \pm 74$ \\
\hline $0301+19$ & 4.85 & $94.3 \pm 15.0$ & $-13.9 \pm 15.0$ & $1416 \pm 888$ \\
\hline $0329+54$ & 10.45 & $94.0 \pm 15.0$ & $-12.4 \pm 15.0$ & $-23 \pm 192$ \\
\hline \multirow[t]{3}{*}{$0355+54$} & 1.71 & $53.1 \pm 80.0$ & $-8.6 \pm 4.0$ & $303 \pm 76$ \\
\hline & 4.85 & $155.8 \pm 80.0$ & $-2.7 \pm 4.0$ & $214 \pm 120$ \\
\hline & 10.45 & $111.9 \pm 80.0$ & $-10.2 \pm 6.0$ & $128 \pm 211$ \\
\hline \multirow[t]{3}{*}{$0525+21$} & 1.41 & $135.8 \pm 60.0$ & $1.3 \pm .2$ & $224 \pm 31$ \\
\hline & 1.71 & $66.3 \pm 80.0$ & $2.0 \pm 1.0$ & $736 \pm 467$ \\
\hline & & $17.8 \pm 70.0$ & $0.6 \pm 2.0$ & $378 \pm 174$ \\
\hline \multirow[t]{2}{*}{$0535+28$} & 1.41 & $108.5 \pm 30.0$ & $-11.5 \pm 6.0$ & $619 \pm 96$ \\
\hline & 4.85 & $79.5 \pm 15.0$ & $-18.5 \pm 15.0$ & $240 \pm 267$ \\
\hline \multirow[t]{3}{*}{$0540+23$} & 1.41 & $162.5 \pm 80.0$ & $-3.5 \pm 6.0$ & $612 \pm 81$ \\
\hline & 4.85 & $80.3 \pm 20.0$ & $10.0 \pm 10.0$ & $730 \pm 155$ \\
\hline & 10.45 & $101.1 \pm 90.0$ & $-8.3 \pm 6.0$ & $297 \pm 167$ \\
\hline \multirow[t]{3}{*}{$0740-28$} & 1.41 & $96.2 \pm 20.0$ & $-16.0 \pm 15.0$ & $210 \pm 29$ \\
\hline & 4.85 & $78.2 \pm 15.0$ & $14.8 \pm 15.0$ & $268 \pm 38$ \\
\hline & 10.45 & $47.3 \pm 90.0$ & $-6.9 \pm 5.0$ & $225 \pm 218$ \\
\hline \multirow{2}{*}{$0809+74$} & 1.71 & $93.4 \pm 10.0$ & $-9.8 \pm 15.0$ & $1267 \pm 438$ \\
\hline & 4.85 & $174.3 \pm 90.0$ & $-0.7 \pm 7.5$ & $447 \pm 725$ \\
\hline \multirow{3}{*}{$0823+26$} & 1.41 & $89.8 \pm 25.0$ & $1.0 \pm 0.2$ & $37 \pm 53$ \\
\hline & 1.71 & $82.3 \pm 80.0$ & $1.7 \pm 1.5$ & $107 \pm 67$ \\
\hline & 4.85 & $97.5 \pm 15.0$ & $-12.1 \pm 15.0$ & $80 \pm 21$ \\
\hline \multirow[t]{2}{*}{$0919+06$} & 1.71 & $90.4 \pm 80.0$ & $2.9 \pm 2.0$ & $314 \pm 87$ \\
\hline & 4.85 & $48.0 \pm 80.0$ & $2.9 \pm 2.5$ & $247 \pm 68$ \\
\hline \multirow[t]{2}{*}{$0950+08$} & 1.41 & $-0.7 \pm 15.0$ & $1.0 \pm 15.0$ & $432 \pm 249$ \\
\hline & 4.85 & $-26.9 \pm 90.0$ & $4.1 \pm 5.0$ & $159 \pm 348$ \\
\hline \multirow[t]{3}{*}{$1133+16$} & 1.41 & $26.9 \pm 90.0$ & $2.8 \pm 4.4$ & $452 \pm 102$ \\
\hline & 1.71 & $103.6 \pm 80.0$ & $4.1 \pm 2.5$ & $505 \pm 146$ \\
\hline & 4.85 & $33.0 \pm 80.0$ & $3.1 \pm 4.0$ & $426 \pm 165$ \\
\hline $1915+13$ & 4.85 & $90.7 \pm 12.5$ & $-9.3 \pm 15.0$ & $346 \pm 75$ \\
\hline $1946+35$ & 4.85 & $52.9 \pm 80.0$ & $4.2 \pm 3.0$ & $133 \pm 168$ \\
\hline $2016+28$ & 4.85 & $89.8 \pm 15.0$ & $4.2 \pm 15.0$ & $159 \pm 181$ \\
\hline \multirow[t]{4}{*}{$2021+51$} & 1.41 & $22.5 \pm 90.0$ & $1.2 \pm 2.5$ & $787 \pm 42$ \\
\hline & 1.71 & $21.0 \pm 80.0$ & $3.1 \pm 5.0$ & $450 \pm 91$ \\
\hline & 4.85 & $22.4 \pm 90.0$ & $4.0 \pm 5.0$ & $389 \pm 137$ \\
\hline & 10.45 & $87.9 \pm 80.0$ & $5.8 \pm 3.0$ & $590 \pm 182$ \\
\hline $2045-16$ & 4.85 & $89.5 \pm 15.0$ & $-1.5 \pm 15.0$ & $443 \pm 232$ \\
\hline $2154+40$ & 4.85 & $101.1 \pm 15.0$ & $-16.0 \pm 15.0$ & $904 \pm 470$ \\
\hline \multirow[t]{2}{*}{$2319+40$} & 1.41 & $102.3 \pm 80.0$ & $-8.1 \pm 4.0$ & $-418 \pm 223$ \\
\hline & 4.85 & $89.9 \pm 15.0$ & $-6.5 \pm 15.0$ & $-1242 \pm 540$ \\
\hline $2351+61$ & 1.41 & $61.2 \pm 80.0$ & $-2.9 \pm 2.0$ & $379 \pm 241$ \\
\hline
\end{tabular}

the linear polarization was $3 \sigma$ above the noise level. In certain cases where significant off-pulse emission was detected, this criterion was relaxed. When a fit of the RVM to the data was applied, the fitted function was overplotted to the data as a solid line. The points which were considered for these fits are marked with circles.

\section{Conclusions}

Carefully conducted polarimetric observations still suffer from polarization state changes due to gain and phase delays, cross-coupling, and depolarization in the observing 
Table 3. The polarization parameters for each frequency for the pulsars in our sample

\begin{tabular}{|c|c|c|c|c|c|c|c|}
\hline Pulsar & $\begin{array}{l}\text { Freq. } \\
{[\mathrm{GHz}]}\end{array}$ & $\begin{array}{l}\text { Cut-level } \\
{[\%]}\end{array}$ & $\begin{array}{l}\text { Width } \\
\left.{ }^{\circ}\right]\end{array}$ & $\begin{array}{l}\text { Linear } \\
{[\%]}\end{array}$ & $\begin{array}{l}\text { Circular } \\
{[\%]}\end{array}$ & $\begin{array}{l}\text { Time } \\
{[\mathrm{min}]}\end{array}$ & $\begin{array}{l}\text { Flux } \\
{[\mathrm{mJy}]}\end{array}$ \\
\hline PSR B0136+57 & 4.85 & 10 & $12.5 \pm 2.6$ & $54.0 \pm 1.0$ & $12.1 \pm 1.5$ & 20 & 0.17 \\
\hline PSR B0301+19 & 4.85 & 10 & $14.0 \pm 1.6$ & $10.3 \pm 3.0$ & $5.5 \pm 5.0$ & 45 & 3.09 \\
\hline \multirow[t]{4}{*}{ PSR B0329+54 } & 1.41 & 2 & $30.7 \pm 1.1$ & $24.1 \pm 0.1$ & $36.3 \pm 0.1$ & 10 & 90.9 \\
\hline & 1.71 & 7 & $28.8 \pm 1.2$ & $21.0 \pm 0.0$ & $9.3 \pm 0.0$ & 10 & 39.4 \\
\hline & 4.85 & 7.6 & $27.4 \pm 0.9$ & $12.3 \pm 0.2$ & $7.4 \pm 0.2$ & 10 & 20.5 \\
\hline & 10.45 & 33 & $22.2 \pm 1.7$ & $12.9 \pm 2.3$ & $7.3 \pm 3.3$ & 15 & 1.75 \\
\hline \multirow[t]{3}{*}{ PSR B0355+54 } & 1.71 & 10 & $35.2 \pm 4.8$ & $53.9 \pm 2.1$ & $4.7 \pm 0.4$ & 1 & 33.4 \\
\hline & 4.85 & 5 & $33.5 \pm 6.0$ & $69.3 \pm 1.3$ & $8.2 \pm 1.0$ & 10 & 3.36 \\
\hline & 10.45 & 5 & $31.4 \pm 21.7$ & $41.9 \pm 5.5$ & $0.9 \pm 5.7$ & 15 & 3.37 \\
\hline \multirow{2}{*}{ PSR B0450+55 } & 1.41 & 5 & $33.5 \pm 3.0$ & $35.2 \pm 0.9$ & $11.0 \pm 1.4$ & 5 & 21.9 \\
\hline & 4.85 & 10 & $29.3 \pm 6.1$ & $44.8 \pm 1.5$ & $6.0 \pm 1.6$ & 15 & 1.78 \\
\hline \multirow[t]{3}{*}{ PSR B0525+21 } & 1.41 & 1 & $21.1 \pm 0.3$ & $40.4 \pm 0.2$ & $3.9 \pm 0.3$ & 3 & 85.7 \\
\hline & 1.71 & 10 & $17.9 \pm 0.7$ & $30.9 \pm 1.5$ & $3.4 \pm 1.1$ & 10 & 8.8 \\
\hline & 4.85 & 3 & $17.2 \pm 0.4$ & $20.6 \pm 0.6$ & $1.0 \pm 0.9$ & 90 & 0.10 \\
\hline \multirow[t]{2}{*}{ PSR B0535+28 } & 1.41 & 15.9 & $29.7 \pm 4.5$ & $82.9 \pm 1.7$ & $1.0 \pm 0.9$ & 13 & 3.1 \\
\hline & 4.85 & 16.9 & $31.9 \pm 11.3$ & $51.1 \pm 2.8$ & $1.0 \pm 0.9$ & 15 & 0.94 \\
\hline \multirow[t]{3}{*}{ PSR B0540+23 } & 1.41 & 3 & $33.7 \pm 4.9$ & $46.9 \pm 0.9$ & $3.8 \pm 0.4$ & 3 & 22.2 \\
\hline & 4.85 & 7 & $28.4 \pm 4.9$ & $29.7 \pm 1.2$ & $13.4 \pm 1.3$ & 20 & 2.19 \\
\hline & 10.45 & 10 & $22.5 \pm 4.2$ & $6.2 \pm 7.1$ & $3.6 \pm 7.6$ & 35 & 1.16 \\
\hline \multirow[t]{3}{*}{ PSR B0740-28 } & 1.41 & 4 & $19.6 \pm 2.1$ & $77.0 \pm 0.5$ & $10.7 \pm 0.5$ & 5 & 20.7 \\
\hline & 4.85 & 7 & $20.3 \pm 2.0$ & $63.4 \pm 1.7$ & $8.5 \pm 1.9$ & 60 & 2.47 \\
\hline & 10.45 & 10 & $14.2 \pm 8.8$ & $39.9 \pm 12.2$ & $4.3 \pm 12.9$ & 30 & 0.65 \\
\hline \multirow{2}{*}{ PSR B0809+74 } & 1.71 & 8 & $29.0 \pm 3.4$ & $23.4 \pm 2.5$ & $9.1 \pm 2.6$ & 8 & 10.7 \\
\hline & 4.85 & 10 & $26.7 \pm 3.6$ & $14.1 \pm 1.0$ & $3.1 \pm 1.6$ & 15 & 2.54 \\
\hline \multirow[t]{3}{*}{ PSR B0823+26 } & 1.41 & 5 & $9.3 \pm 1.1$ & $20.0 \pm 3.1$ & $3.4 \pm 2.7$ & 3 & 12.8 \\
\hline & 1.71 & 5 & $8.0 \pm 1.4$ & $22.8 \pm 5.9$ & $2.8 \pm 6.9$ & 10 & 4.1 \\
\hline & 4.85 & 1 & $13.8 \pm 1.6$ & $17.3 \pm 0.9$ & $0.7 \pm 1.4$ & 30 & 2.63 \\
\hline \multirow[t]{2}{*}{ PSR B0919+06 } & 1.71 & 8 & $10.5 \pm 2.0$ & $38.5 \pm 10.6$ & $27.5 \pm 13.5$ & 5 & 3.2 \\
\hline & 4.85 & 7 & $7.9 \pm 1.4$ & $47.3 \pm 2.6$ & $4.6 \pm 3.0$ & 45 & 0.63 \\
\hline \multirow[t]{3}{*}{ PSR B0950+08 } & 1.41 & 1 & $72.4 \pm 4.7$ & $14.8 \pm 0.1$ & $3.7 \pm 0.1$ & 5 & 80.4 \\
\hline & 4.85 & 4 & $45.3 \pm 8.6$ & $8 \pm 0.8$ & $0.3 \pm 1.3$ & 10 & 41.3 \\
\hline & 10.45 & 15 & $23.8 \pm 11.9$ & $5.3 \pm 6.4$ & $3.2 \pm 9.2$ & 50 & 8.61 \\
\hline \multirow{4}{*}{ PSR B1133+16 } & 1.41 & 8 & $10.9 \pm 0.6$ & $15.8 \pm 0.4$ & $1.8 \pm 0.7$ & 5 & 19.5 \\
\hline & 1.71 & 4 & $11.6 \pm 0.3$ & $12.1 \pm 0.2$ & $5.4 \pm 0.3$ & 10 & 24.5 \\
\hline & 4.85 & 8 & $9.4 \pm 0.5$ & $10.4 \pm 0.3$ & $2.4 \pm 0.4$ & 20 & 4.38 \\
\hline & 10.45 & 8 & $9.8 \pm 6.9$ & $7.8 \pm 23.9$ & $10.4 \pm 31.2$ & 20 & 0.45 \\
\hline
\end{tabular}

setup. A detailed analysis is presented here for an adding and a multiplying polarimeter used with three different receivers with the Effelsberg radio telescope to perform high quality polarimetry. The analysis consists of a method to dynamically detect and account for the differential gains in the observing system. The gain monitoring is based on the injection of a calibration signal prior to the polarizer. This signal is synchronous to the pulsar period and is dynamically adjusted for imbalances between the polarization channels if needed. Once gain corrected data are available, a detailed procedure is followed to account for cross-coupling effects. These effects are then removed from the measurements. Using the method we have described, the performance of the observing system can be routinely evaluated. This reduction procedure provided evidence for the stability of the polarization characteristics of the instrumental set-up used, and ensured the high quality of the polarization data. The sample of 28 pulsars at many frequencies presented here was also used to derive emission altitudes based on a method developed by
Blaskiewicz et al. (1991) and the results and interpretation are presented in von Hoensbroech \& Xilouris (1997). The polarization data presented here comprise a reliable database at cm-wavelengths which can facilitate further studies of polarization effects of pulsars at high frequencies.

Acknowledgements. We want to express our gratitude to Drs. M. Kramer, A. Jessner and R. Wielebinski for their constant help, support and valuable advice. We are in particular grateful to C. Salter for extremely helpful suggestions. Arecibo Observatory is operated by Cornell University under cooperative agreement with NSF.

\section{References}

Blaskiewicz M., Cordes J., Wasserman J., 1991, ApJ 370, 643 Cordes J., 1978, ApJ 222, 1006

Hoensbroech A. von, Xilouris K.M., 1996 (accepted)

Kramer M., 1995, PhD-thesis, University of Bonn

Kraus J.D., 1966, Radio Astronomy. McGraw-Hill Book Company, p. 122 
Table 3. continued

\begin{tabular}{llllccll}
\hline Pulsar & $\begin{array}{l}\text { Freq. } \\
{[\mathrm{GHz}]}\end{array}$ & $\begin{array}{l}\text { Cut-level } \\
{[\%]}\end{array}$ & $\begin{array}{l}\text { Width } \\
{\left[{ }^{\circ}\right]}\end{array}$ & $\begin{array}{l}\text { Linear } \\
{[\%]}\end{array}$ & $\begin{array}{l}\text { Circular } \\
{[\%]}\end{array}$ & $\begin{array}{l}\text { Time } \\
{[\mathrm{min}]}\end{array}$ & $\begin{array}{l}\text { Flux } \\
{[\mathrm{mJy}]}\end{array}$ \\
\hline PSR B1237+25 & 1.41 & 2 & $15.4 \pm 0.5$ & $51.0 \pm 0.2$ & $16.0 \pm 0.2$ & 8 & 28.3 \\
& 4.85 & 5 & $13.6 \pm 1.4$ & $13.5 \pm 1.7$ & $5.2 \pm 2.1$ & 20 & 0.79 \\
PSR B1642-03 & 1.41 & 1.6 & $17.9 \pm 3.1$ & $11.2 \pm 1.9$ & $9.2 \pm 2.4$ & 3 & 15.2 \\
& 4.85 & 10 & $18.6 \pm 3.3$ & $8.6 \pm 3.5$ & $1.2 \pm 3.5$ & 25 & 1.10 \\
PSR B1822-09 & 1.41 & 18 & $22.8 \pm 2.2$ & $32.9 \pm 1.1$ & $11.6 \pm 1.2$ & 5 & 6.3 \\
& 4.85 & 10 & $23.5 \pm 1.7$ & $32.1 \pm 1.7$ & $2.3 \pm 0.5$ & 15 & 4.76 \\
& 10.45 & 30 & $19.3 \pm 5.5$ & $86.7 \pm 76.5$ & $36.3 \pm 71.6$ & 25 & 0.26 \\
PSR B1915+13 & 4.85 & 8 & $20.4 \pm 2.6$ & $31.6 \pm 2.5$ & $1.4 \pm 3.6$ & 60 & 0.50 \\
PSR B1929+10 & 1.41 & 1 & $53.8 \pm 4.1$ & $71.9 \pm 0.1$ & $12.5 \pm 0.1$ & 3 & 233.3 \\
& 1.71 & 1 & $52.1 \pm 4.8$ & $71.5 \pm 0.2$ & $13.8 \pm 0.2$ & 2 & 160.6 \\
& 4.85 & 1 & $41.8 \pm 6.0$ & $65.7 \pm 0.5$ & $0.6 \pm 0.5$ & 5 & 6.80 \\
PSR B1946+35 & 10.45 & 10 & $14.8 \pm 3.0$ & $43.1 \pm 4.1$ & $2.9 \pm 5.0$ & 20 & 2.23 \\
PSR B2016+28 & 4.85 & 10 & $18.7 \pm 1.4$ & $12.6 \pm 2.7$ & $0.3 \pm 4.3$ & 20 & 0.50 \\
PSR B2020+28 & 4.85 & 2 & $15.1 \pm 1.9$ & $0.8 \pm 1.7$ & $3.0 \pm 2.1$ & 20 & 0.85 \\
& 10.45 & 8 & $18.3 \pm 0.9$ & $14.3 \pm 0.3$ & $5.7 \pm 0.3$ & 20 & 1.64 \\
PSR B2021+51 & 1.41 & 2 & $13.7 \pm 0.5$ & $5.4 \pm 6.4$ & $8.4 \pm 10.7$ & 45 & 0.77 \\
& 1.71 & 3 & $23.8 \pm 1.1$ & $28.6 \pm 0.1$ & $3.6 \pm 0.1$ & 5 & 52.3 \\
& 4.85 & 3 & $22.2 \pm 2.2$ & $38.8 \pm 0.3$ & $11.0 \pm 0.5$ & 5 & 30.8 \\
PSR B2045-16 & 10.45 & 10 & $19.3 \pm 2.0$ & $22.7 \pm 0.3$ & $0.7 \pm 0.3$ & 25 & 9.73 \\
PSR B2154+40 & 4.85 & 10 & $12.7 \pm 2.6$ & $29.6 \pm 6.3$ & $0.7 \pm 8.3$ & 10 & 1.22 \\
PSR B2310+42 & 1.41 & 5 & $23.2 \pm 0.4$ & $9.2 \pm 1.6$ & $2.2 \pm 2.1$ & 20 & 1.06 \\
& 4.85 & 10 & $17.3 \pm 3.0$ & $25.1 \pm 4.8$ & $0.7 \pm 6.2$ & 20 & 0.64 \\
PSR B2319+60 & 1.41 & 4 & $14.3 \pm 0.9$ & $68.4 \pm 2.0$ & $4.4 \pm 1.6$ & 30 & 12.4 \\
& 4.85 & 5 & $23.3 \pm 0.9$ & $40.1 \pm 0.3$ & $2.7 \pm 1.0$ & 30 & 0.20 \\
PSR B2351+61 & 1.41 & 7 & $20.9 \pm 1.6$ & $21.3 \pm 0.9$ & $9.4 \pm 0.1$ & 8 & 27.1 \\
& 4.85 & 10 & $12.0 \pm 1.4$ & $64.6 \pm 10.0$ & $22.8 \pm 11.3$ & 20 & 0.24 \\
& & $9.5 \pm 3.1$ & $6.8 \pm 3.8$ & $1.8 \pm 4.9$ & 20 & 0.08 \\
\hline
\end{tabular}

Manchester R.N., Taylor J.H., 1977, Pulsars, Freeman, San Francisco

Radhakrishnan V., Cooke D.J., 1969, Astrophys. Lett. 3, 225

Rankin J.M., Campbell D.B., Spangler S.R., 1975, NAICReports, Vol. 44
Saikia D.J., Salter C.J., 1988, ARA\&A 26, 93

Stinebring D.R., Cordes J.M., Weisberg J.M., Rankin J.M., Boriakoff V., 1984, ApJS 55, 27

Turlo S., Fokert T., Sieber W., 1985, A\&A 142, 181

Xilouris K.M., 1991, A\&A 248, 323 
PSR B0136+57
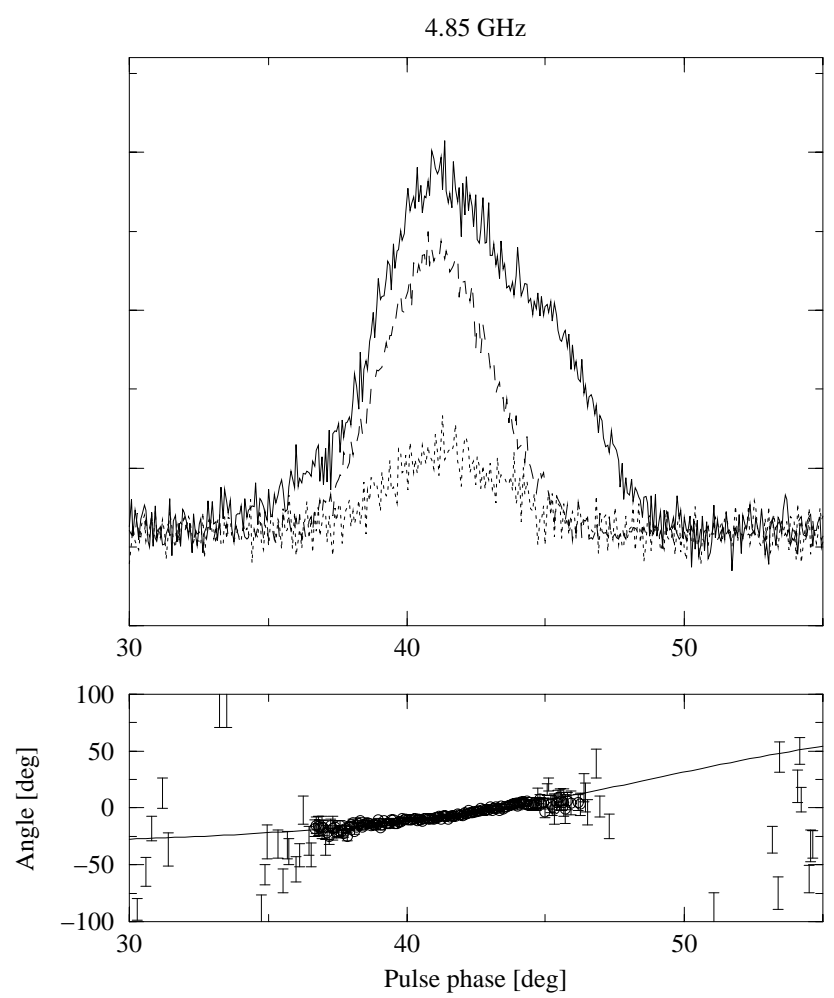

PSR B0301+19
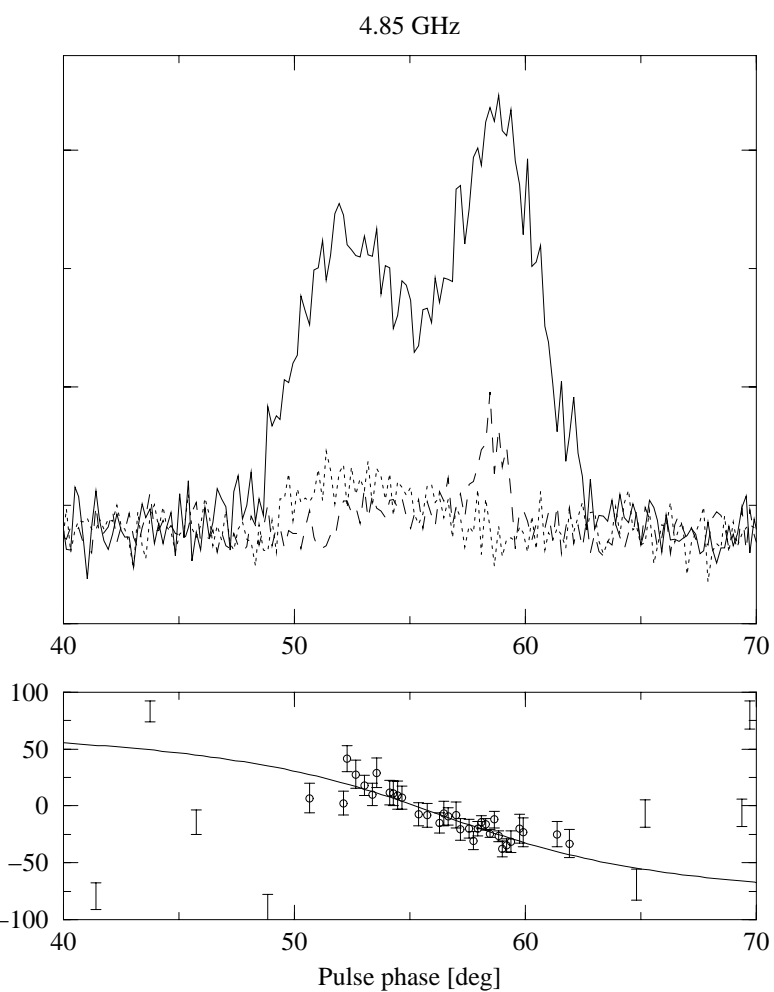

Fig. 5. PSR B0301+57 and PSR B0301+19. For each profile two panels are plotted. The upper one shows total power (solid), linearly- (dashed) and circularly $[V=\mathrm{LHC}-\mathrm{RHC}]$ (dotted) polarized intensity. The lower plot shows the derived PPA-points with their error bars. In all cases only such PPA-points are shown which have a signal-to-noise ratio above a certain level, depending on the polarisation of the pulsar. In those cases when a fit of the RVM to the data was applied, the fitted function is plotted. The points which were considered for the fits are marked with circles 
PSR B0329+54

$1.41 \mathrm{GHz}$
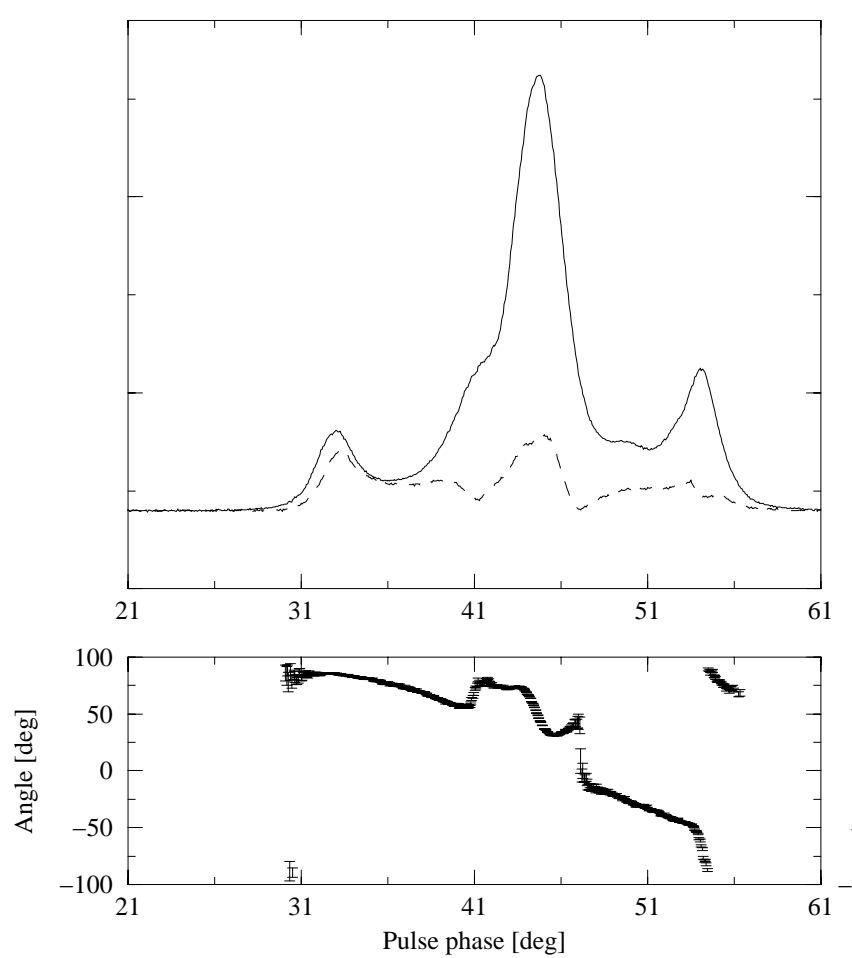

PSR B0329+54
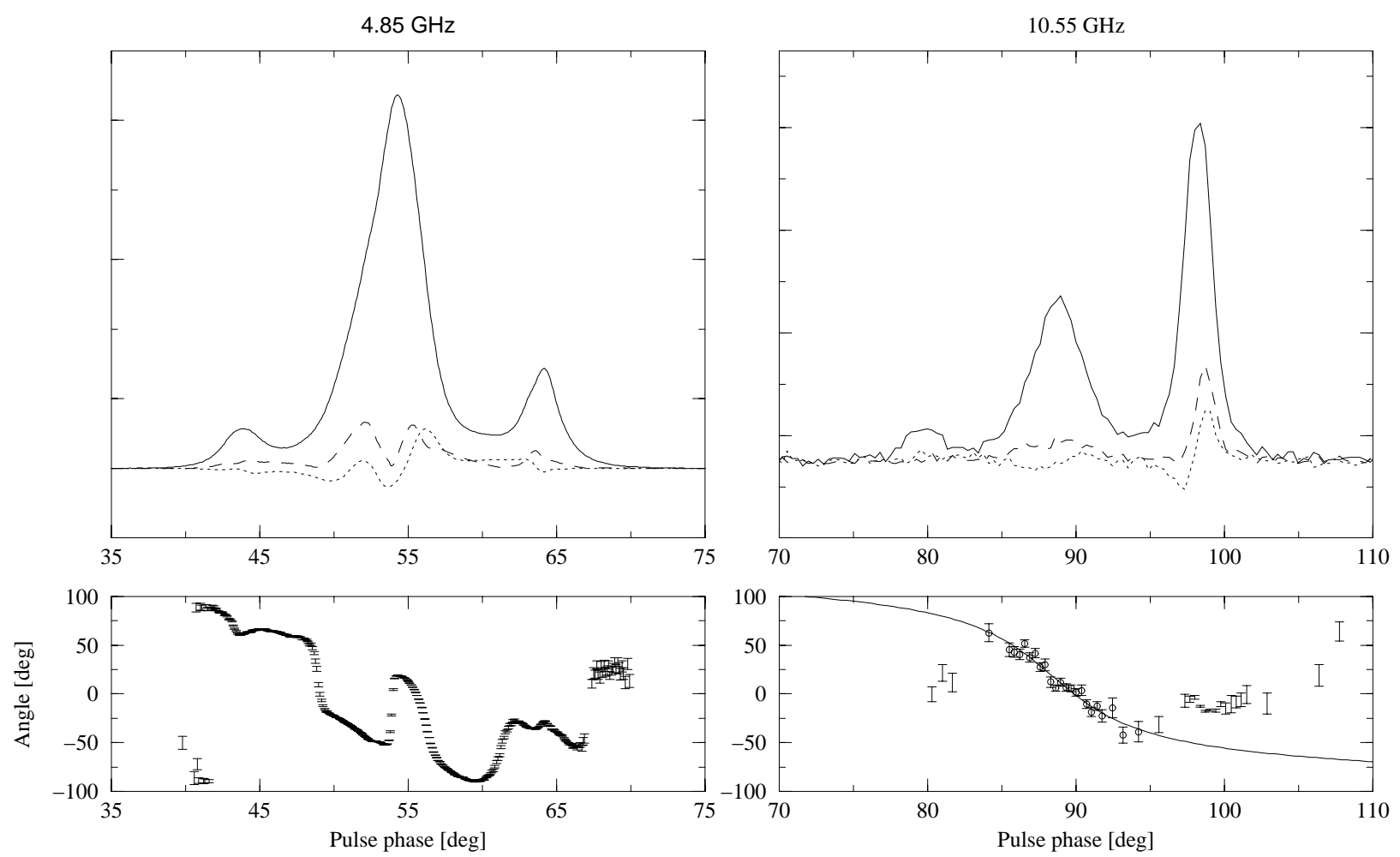

PSR B0329+54
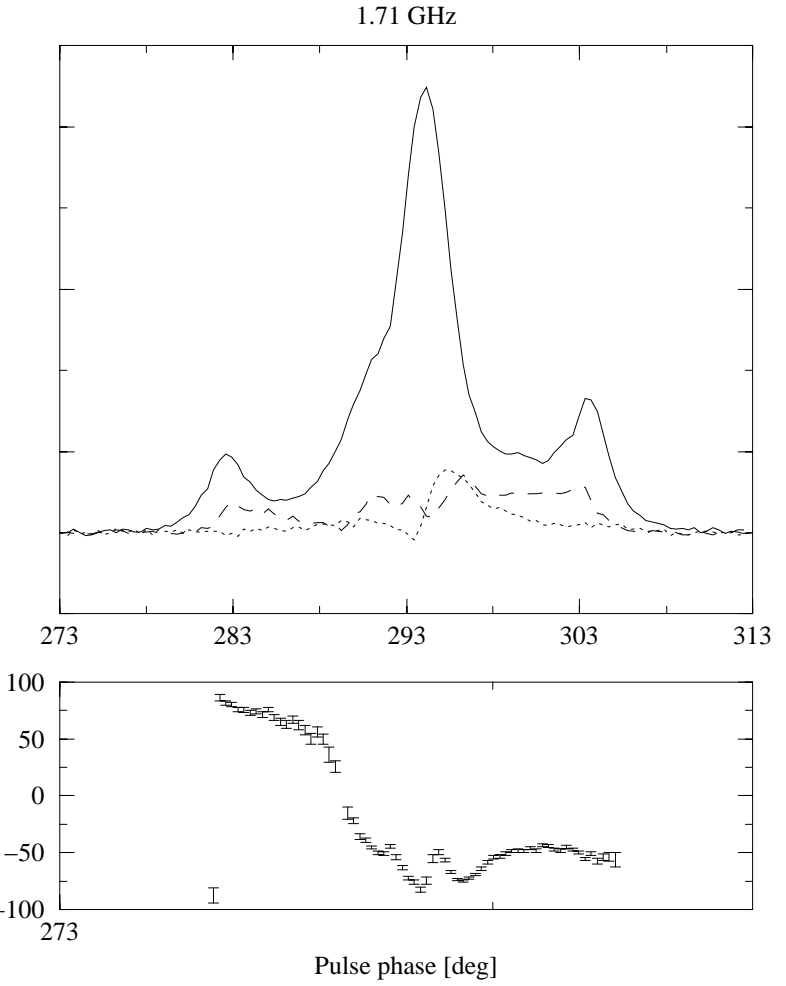

PSR B0329+54

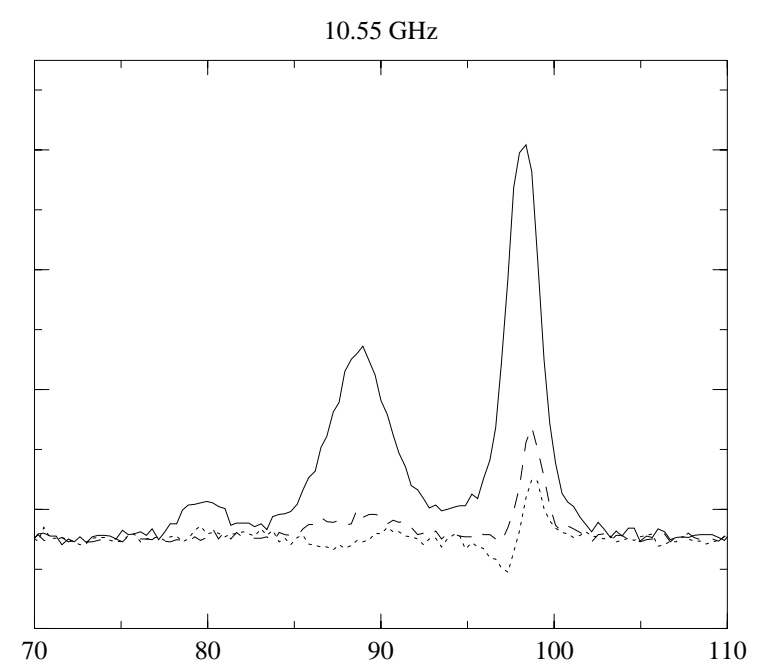

Fig. 6. PSR B0329+54 

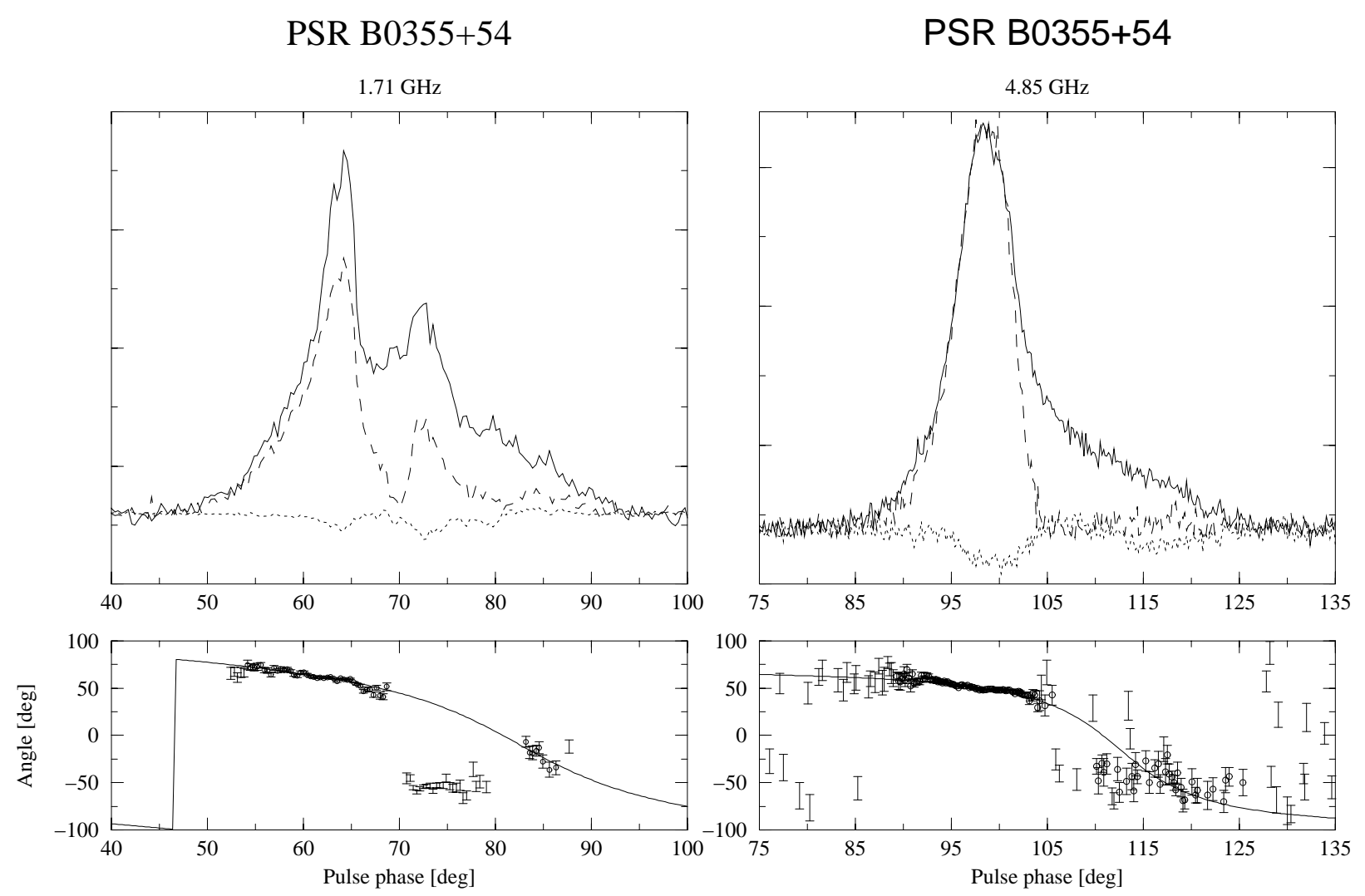

PSR B0355+54
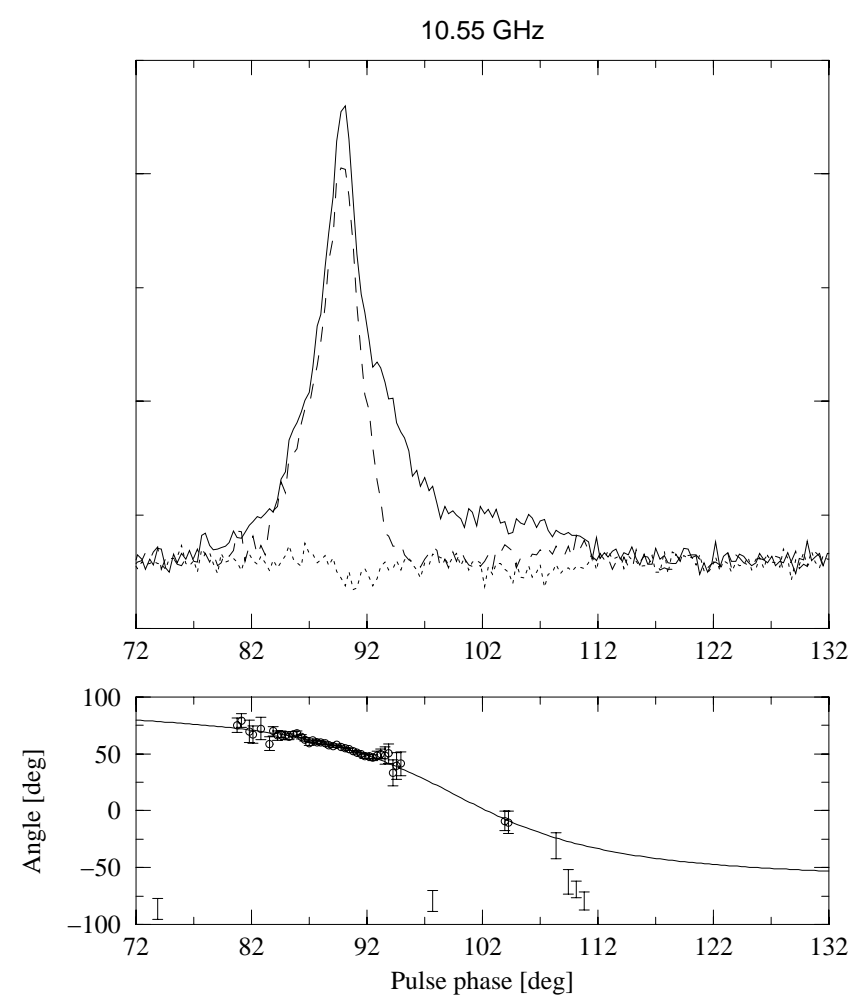

Fig. 7. PSR B0355+54 
PSR B0450+55

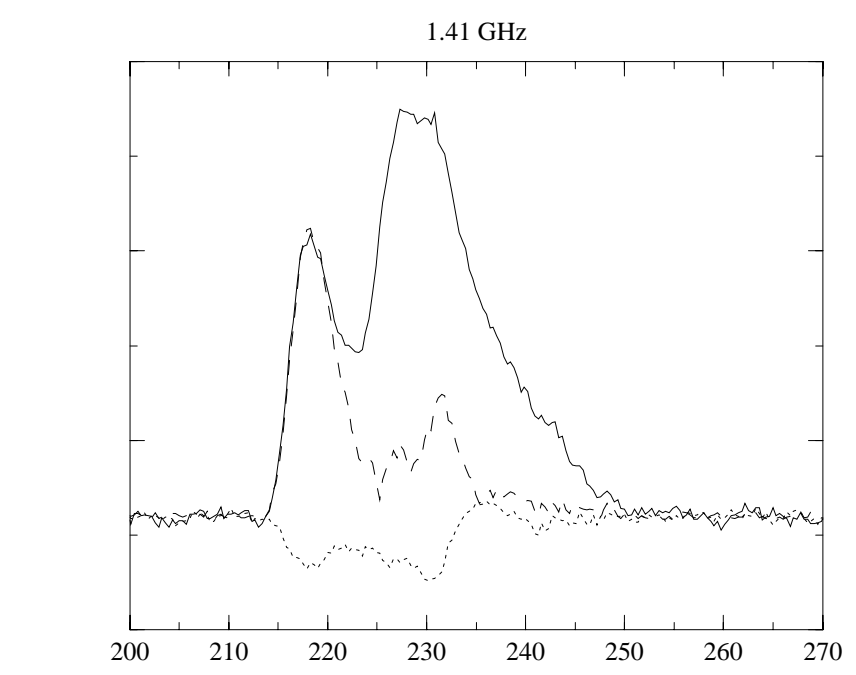

PSR B0450+55
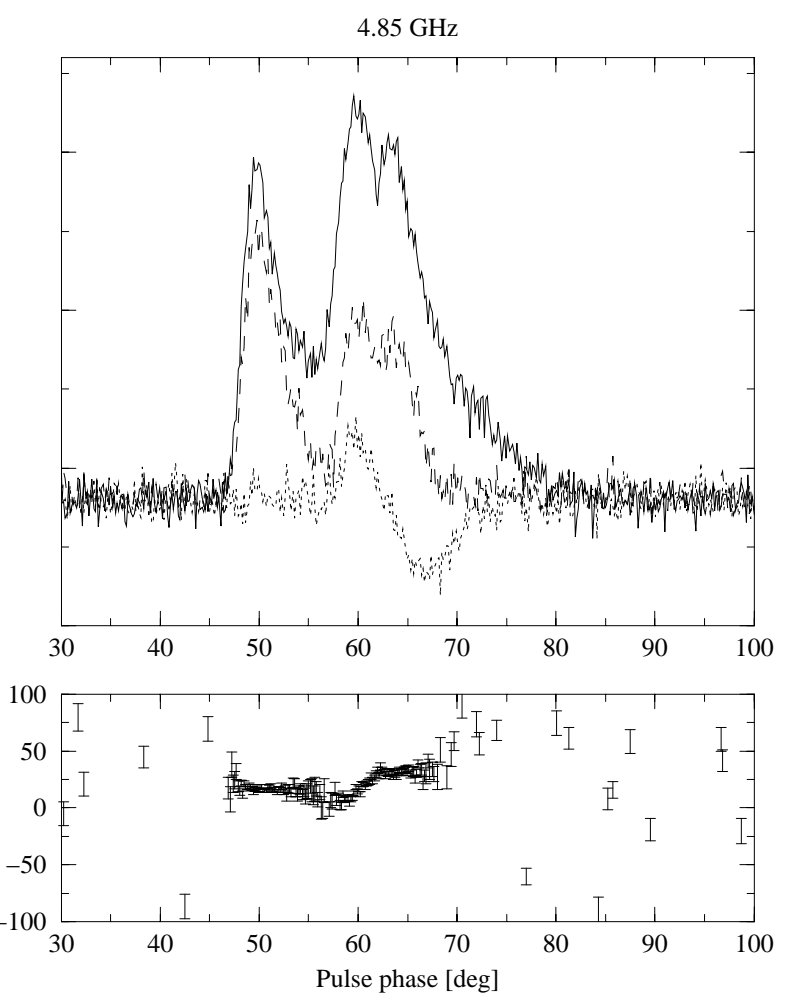

Fig. 8. PSR B0450+55 


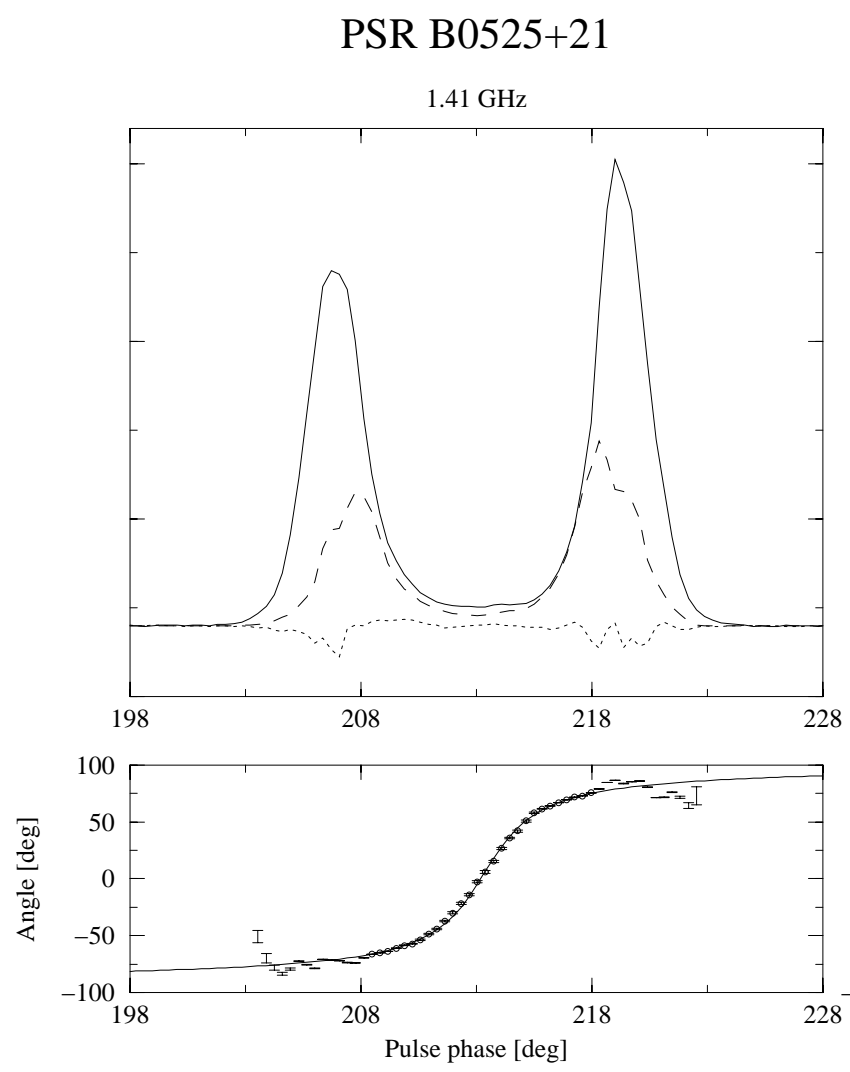

PSR B0525+21
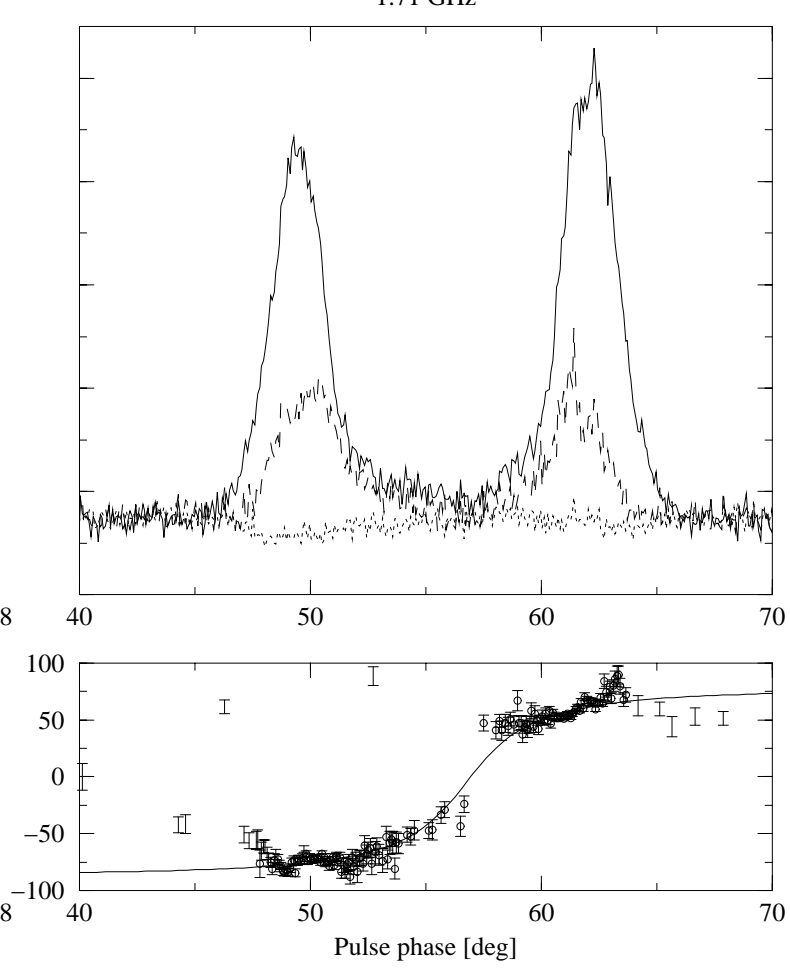

PSR B0525+21
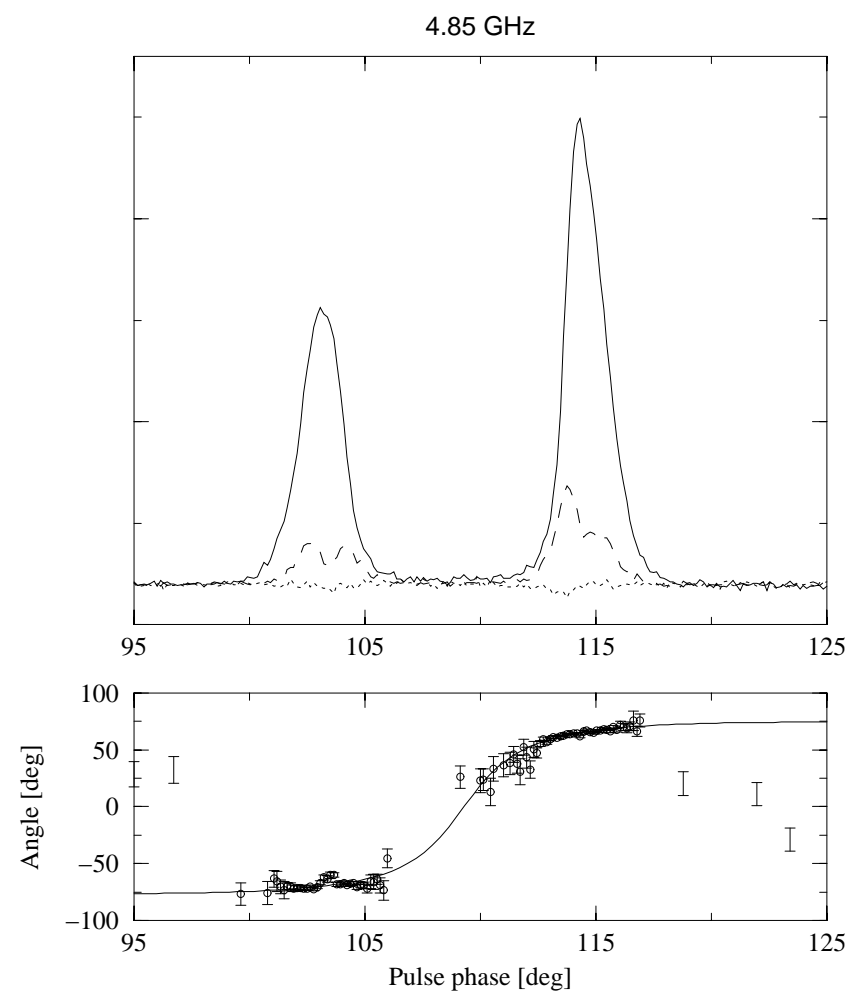

Fig. 9. PSR B $0525+21$ 

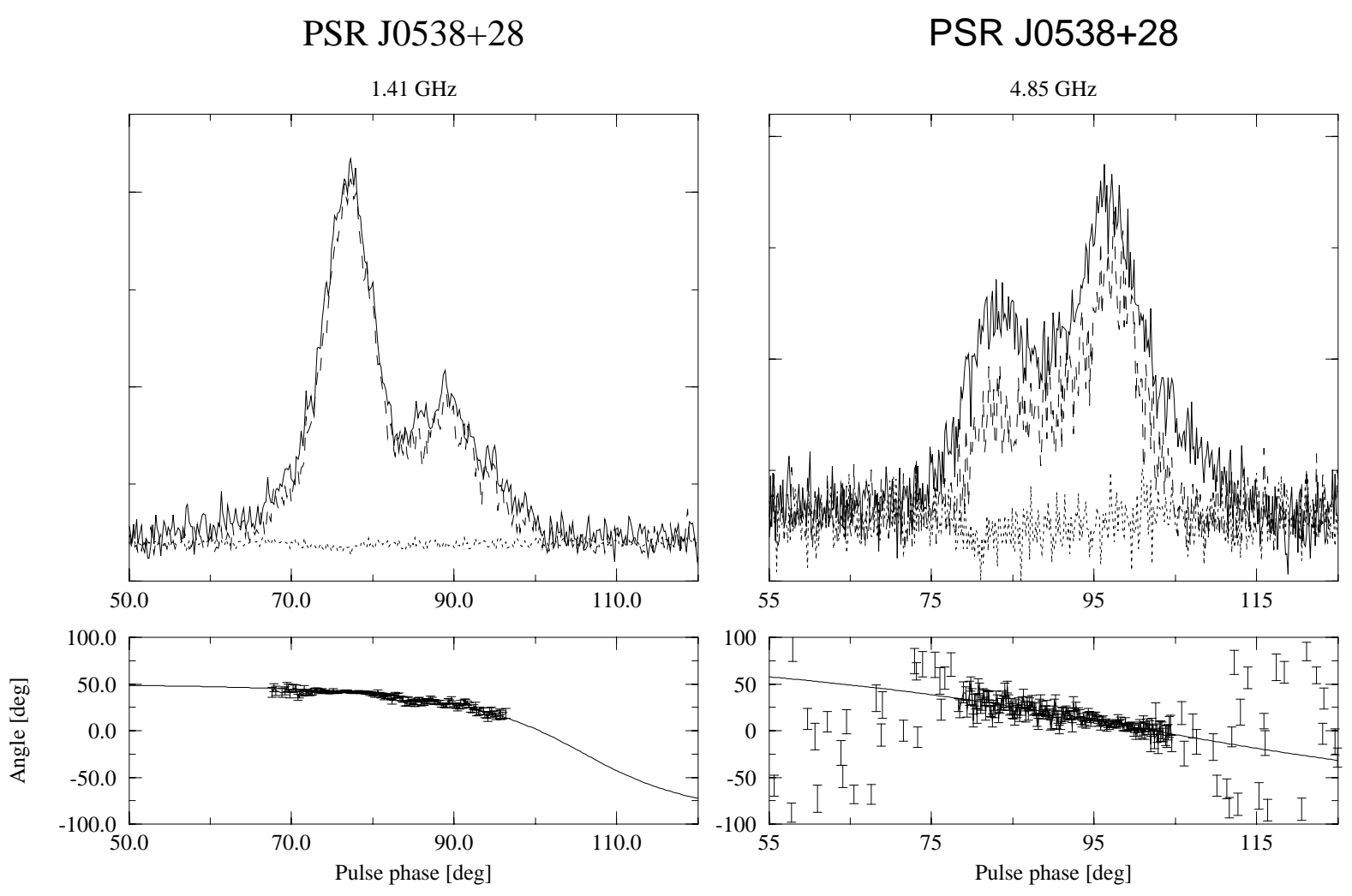

Fig. 10. PSR J0538+28 
PSR B0540+23

$1.41 \mathrm{GHz}$
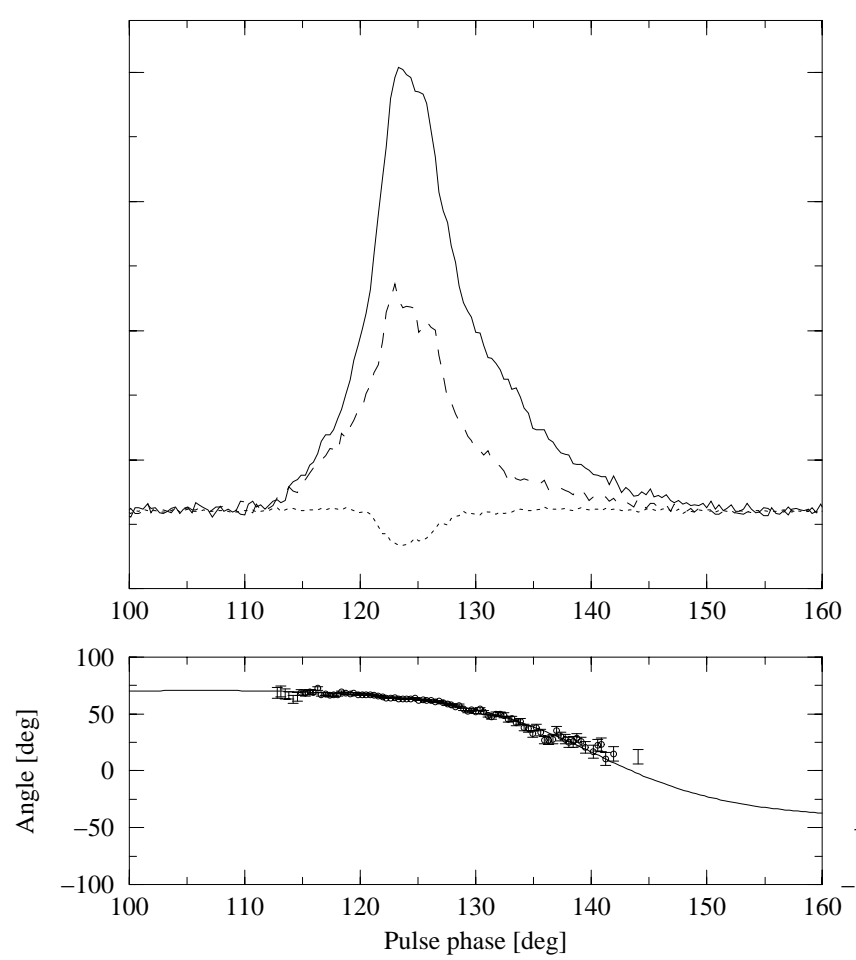

PSR B0540+23
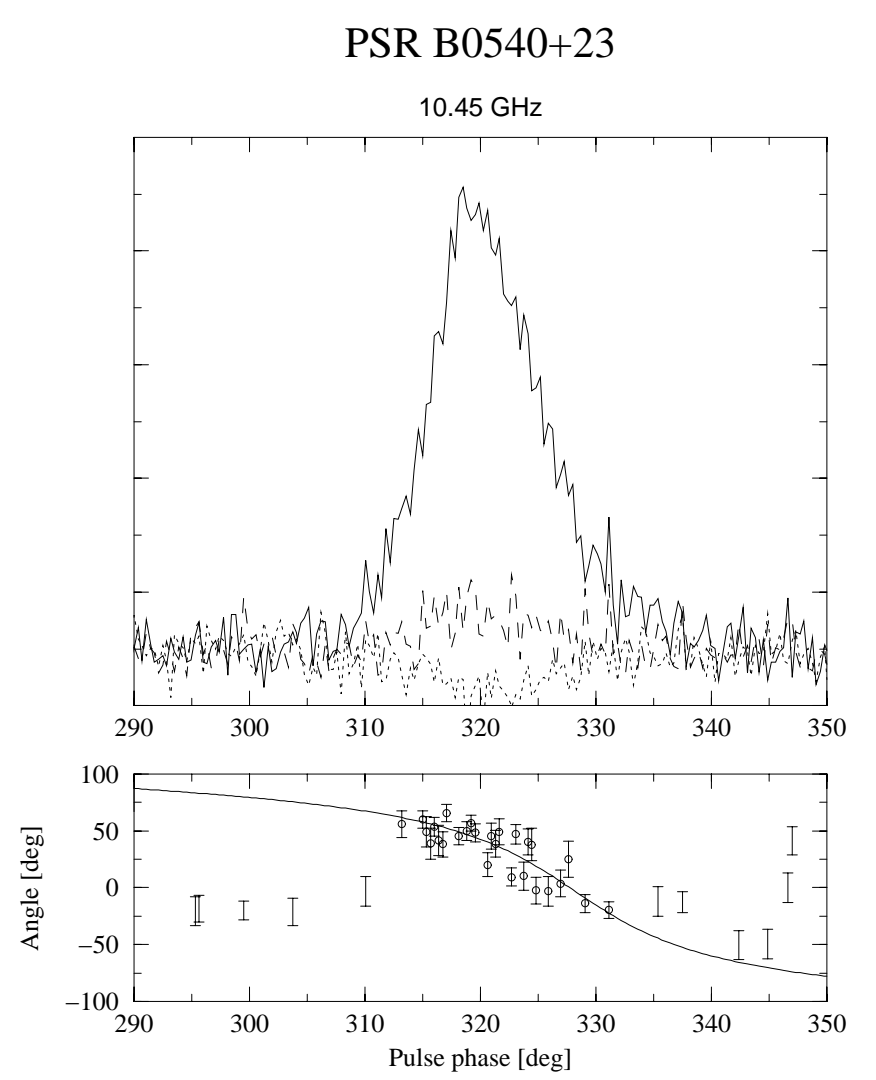

PSR B0540+23
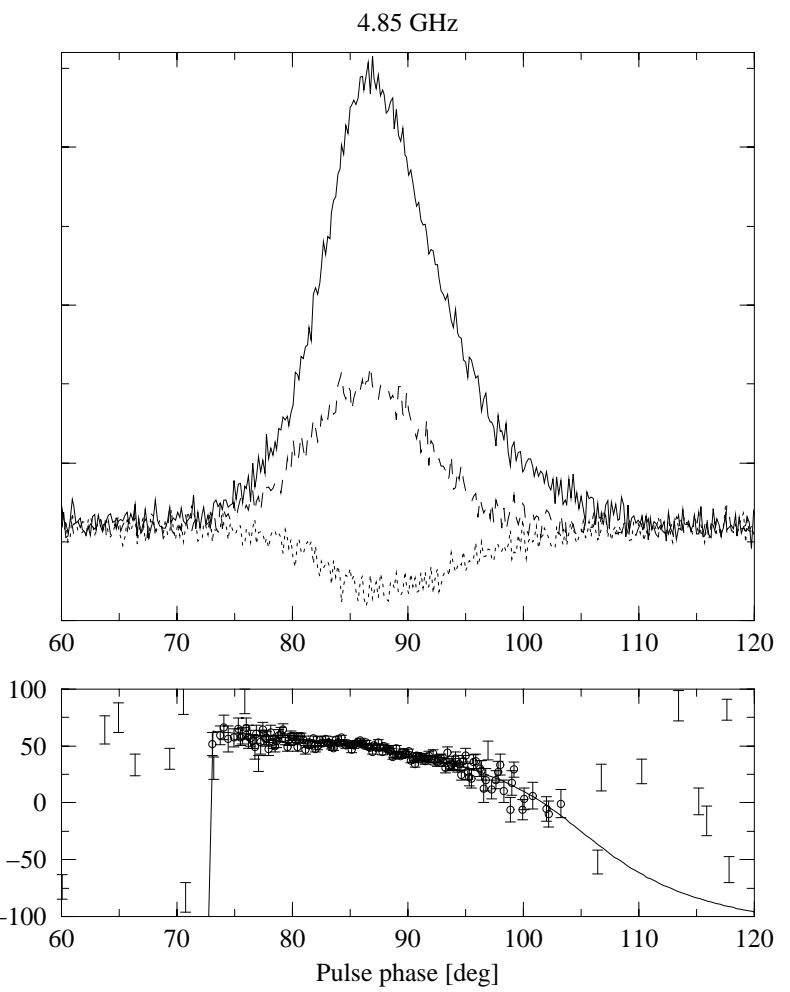

Fig. 11. PSR B0540+23 
PSR B0740-28

$1.41 \mathrm{GHz}$
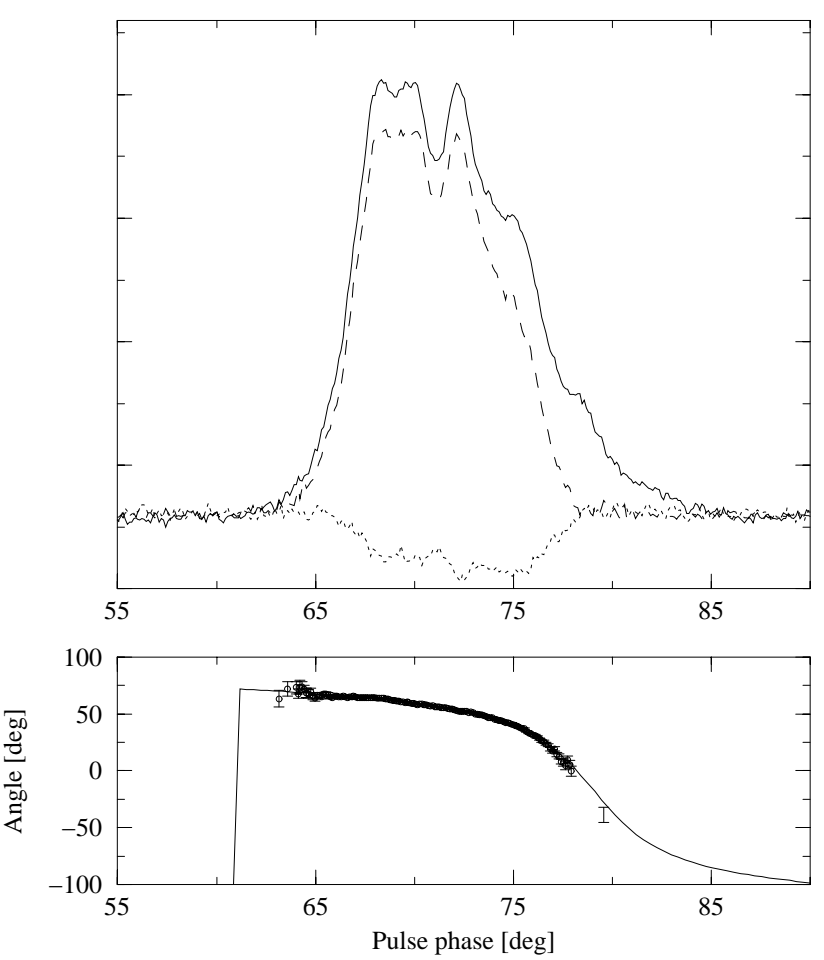

PSR B0740-28
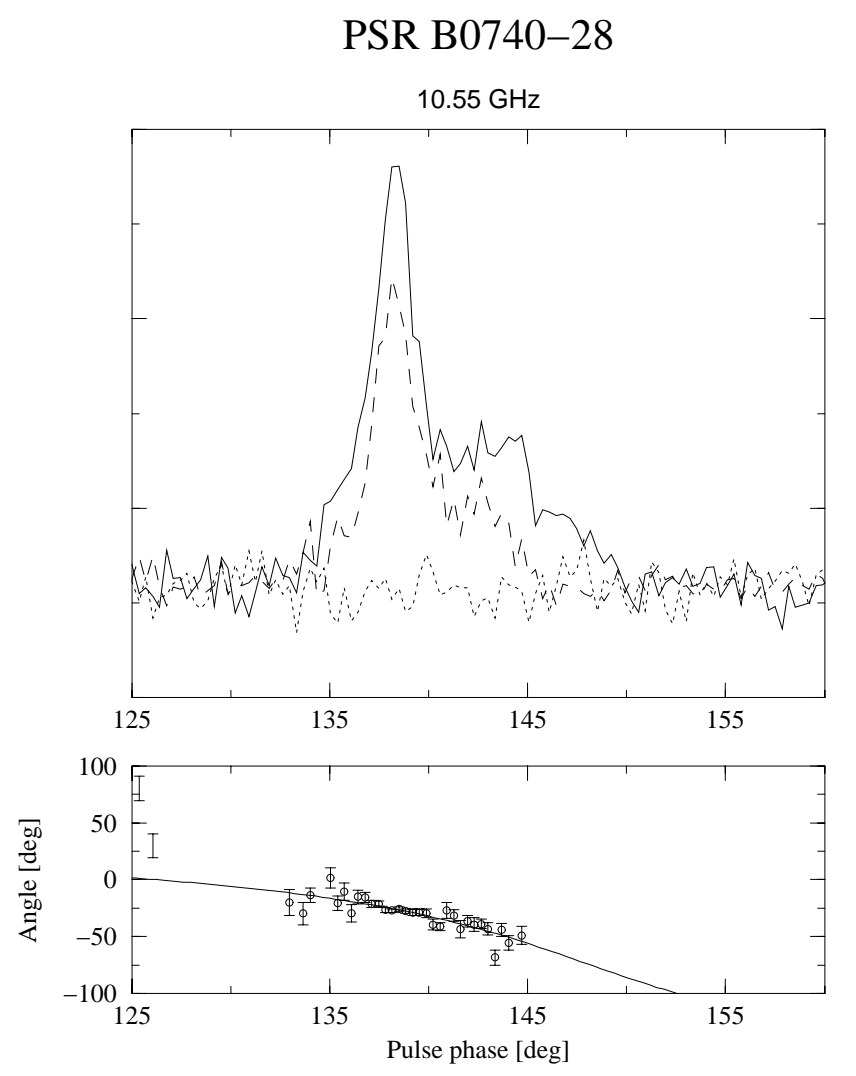

PSR B0740-28
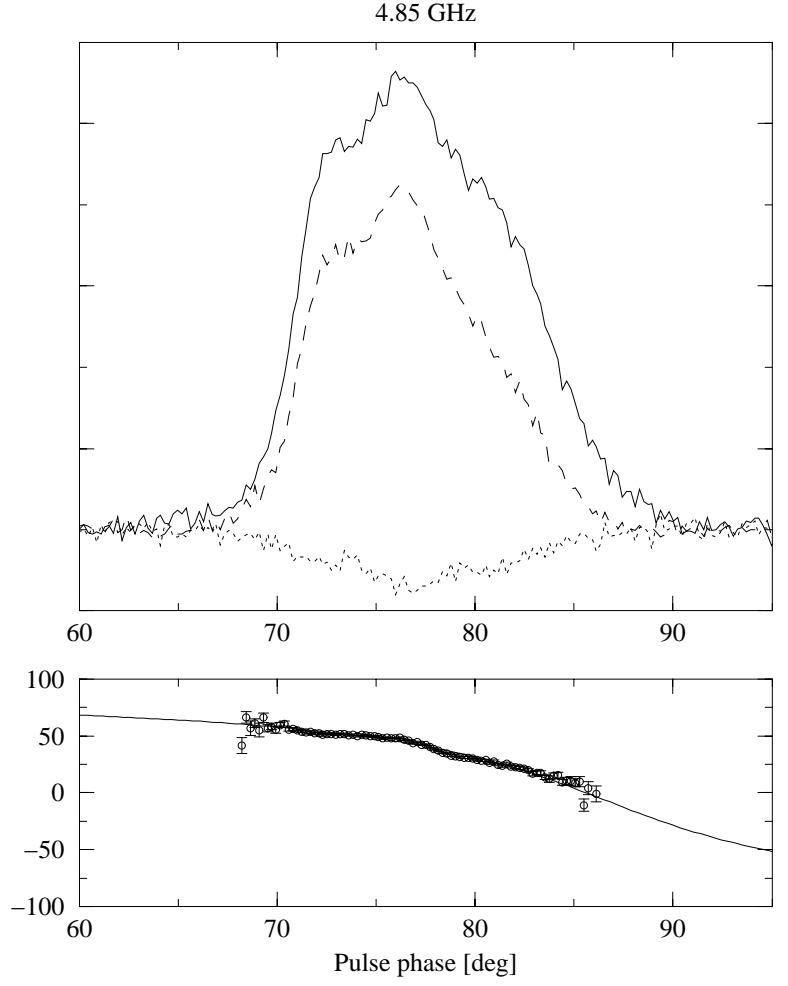

Fig. 12. PSR B0740-28 
PSR B0809+74

$1.41 \mathrm{GHz}$
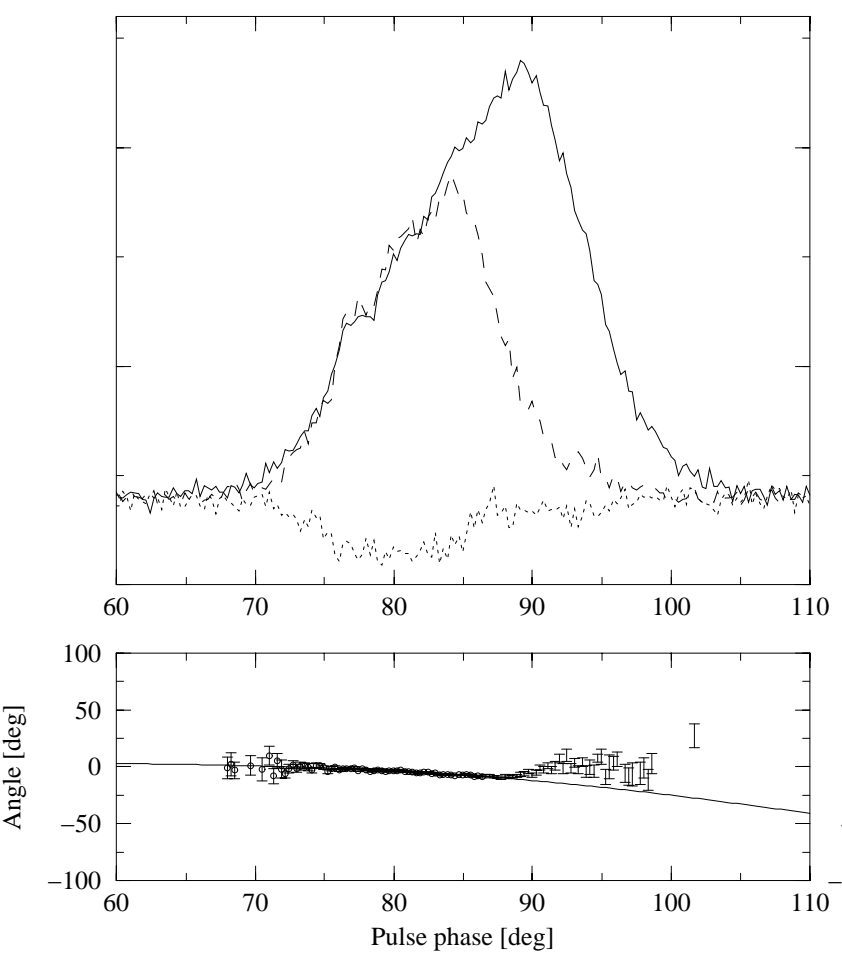

PSR B0809+74
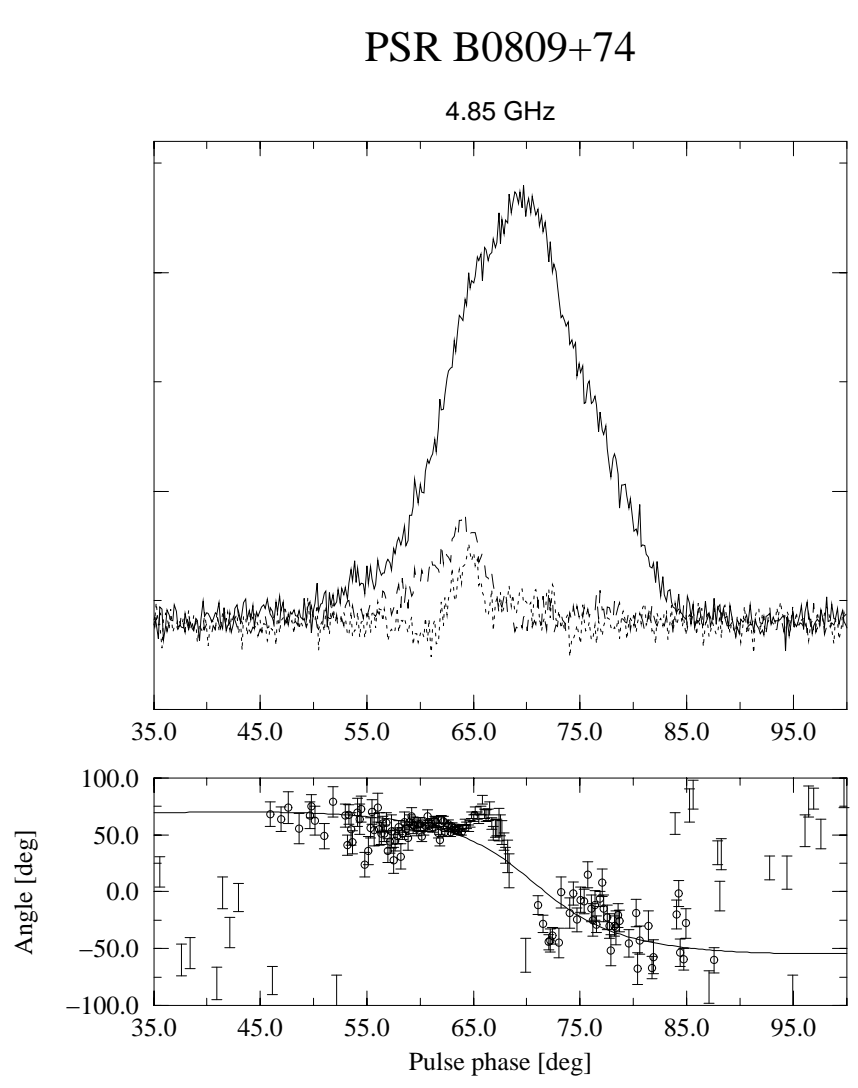

PSR B0809+74
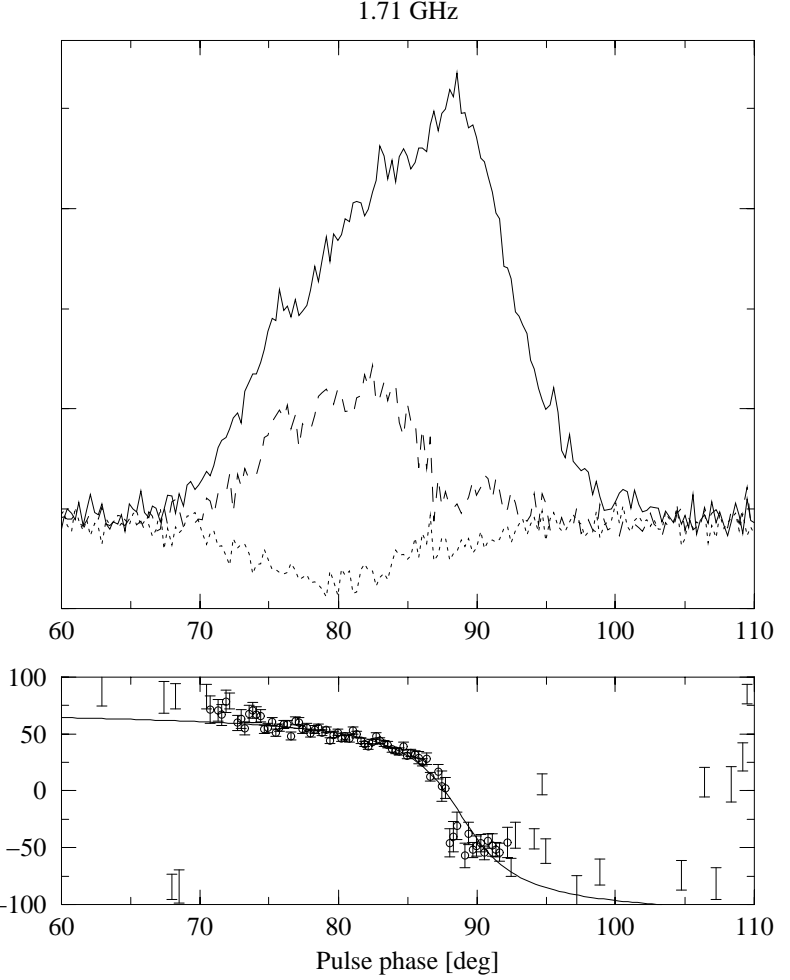

Fig. 13. PSR B0809+74 
PSR B0823+26

$1.41 \mathrm{GHz}$
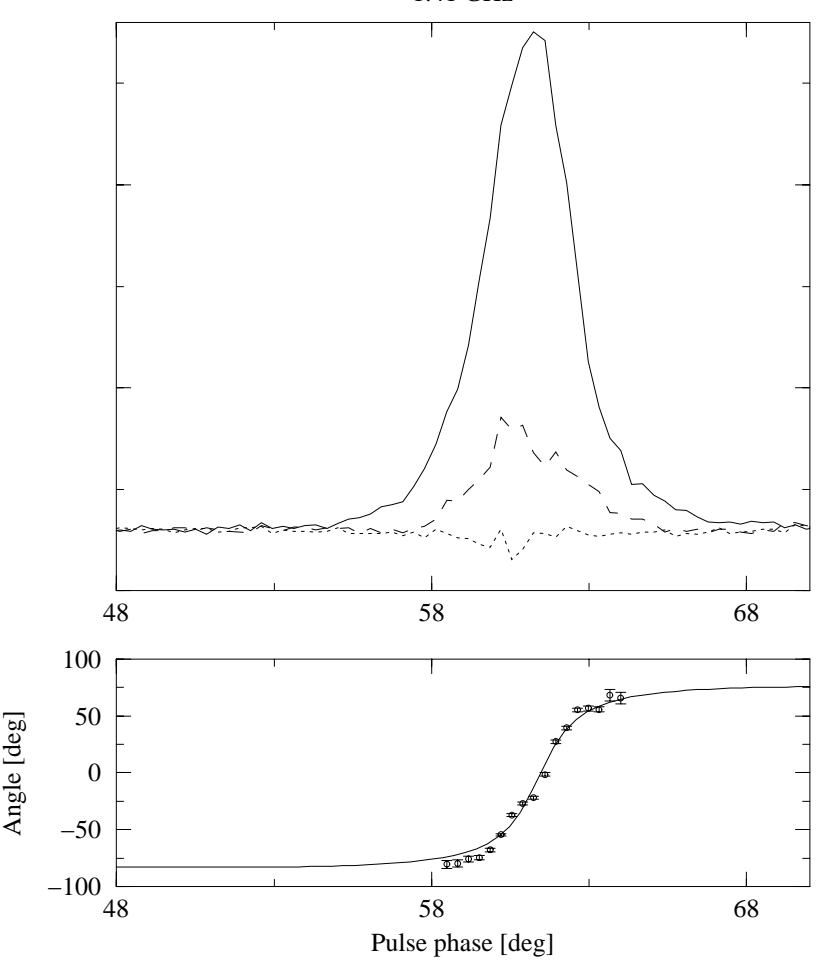

PSR B0823+26
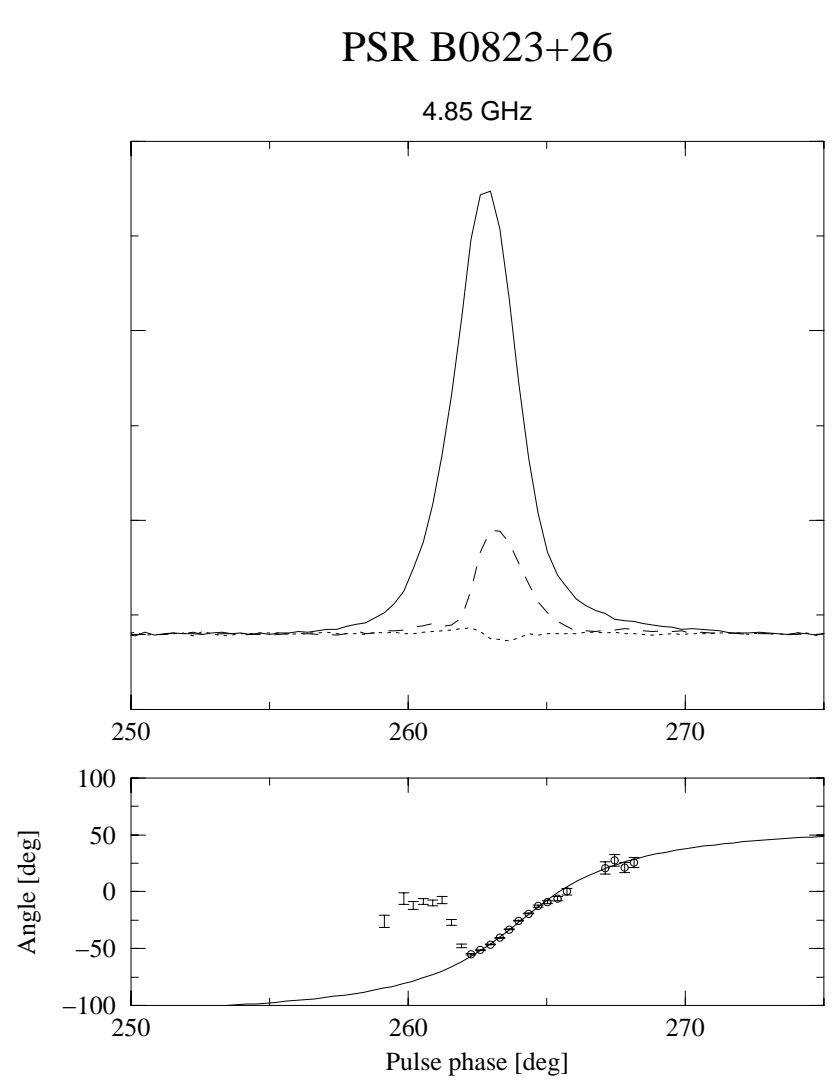

PSR B0823+26
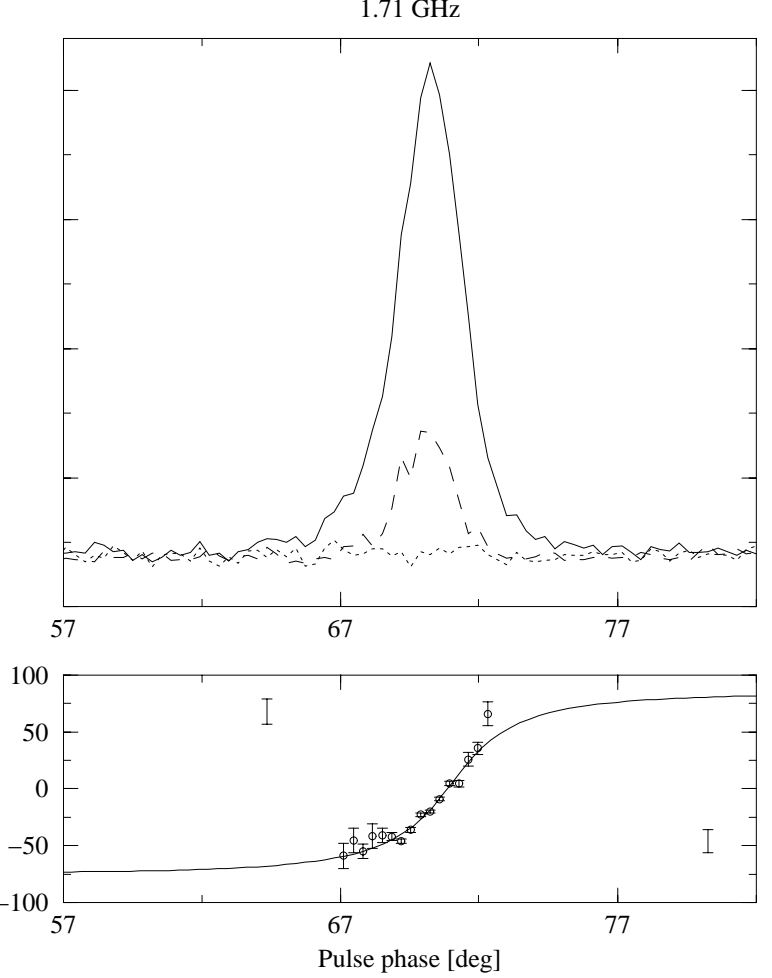

Fig. 14. PSR B $0823+26$ 


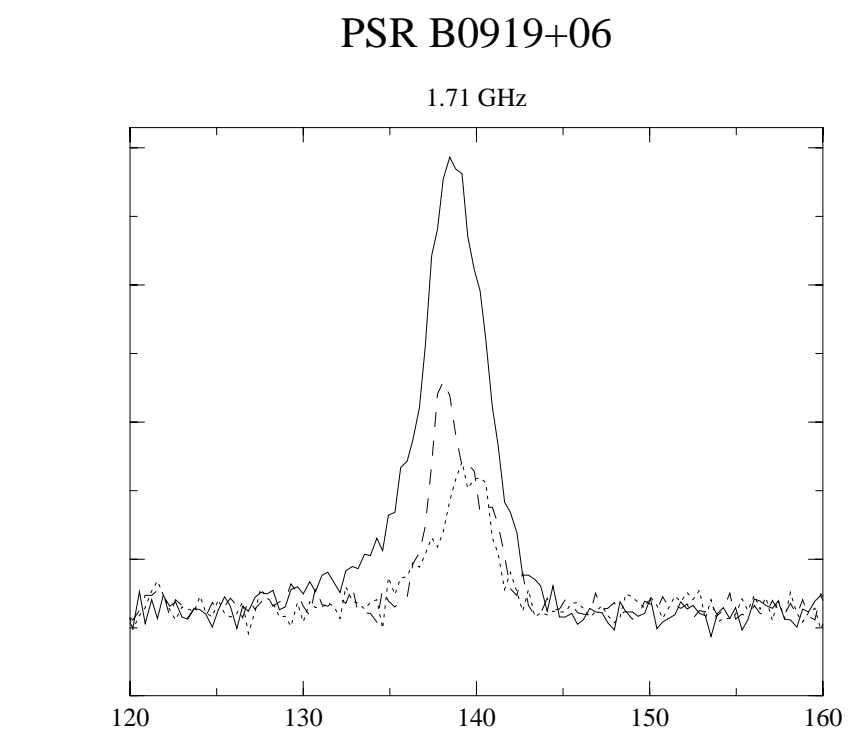

PSR B0919+06
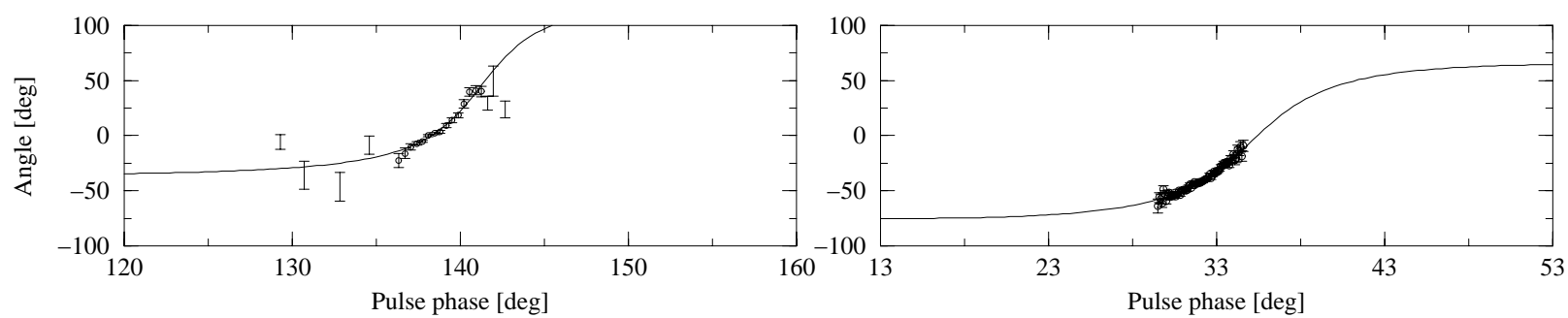

Fig. 15. PSR B0919+06 
PSR B0950+08

$1.41 \mathrm{GHz}$
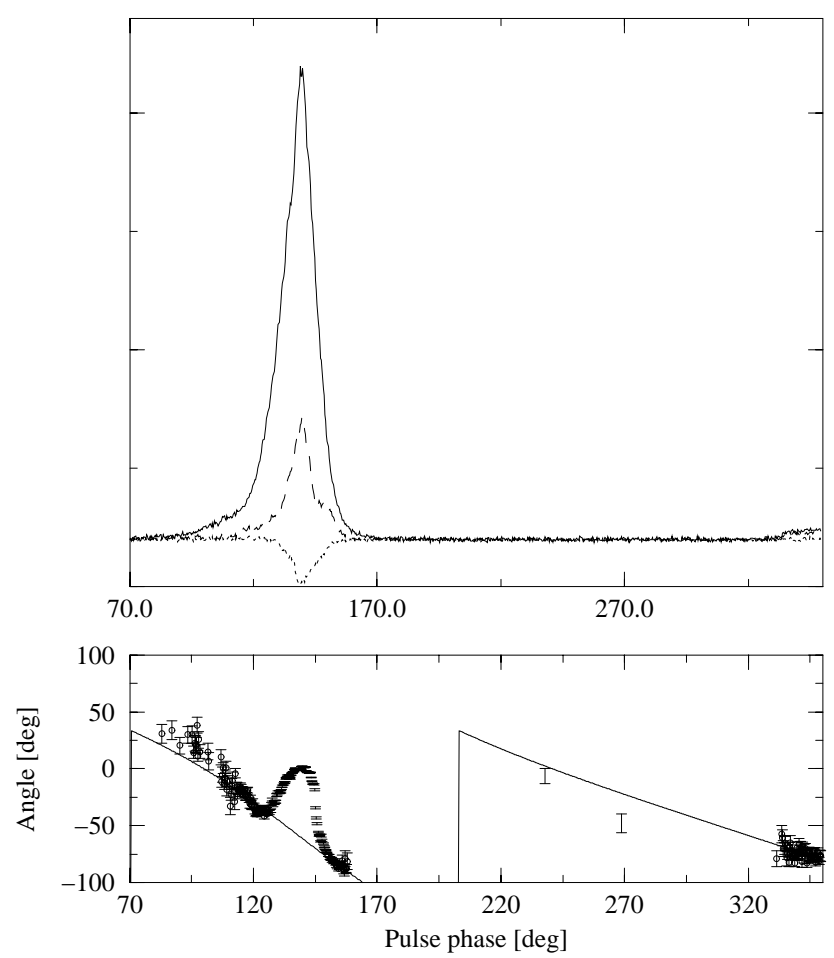

PSR B0950+08
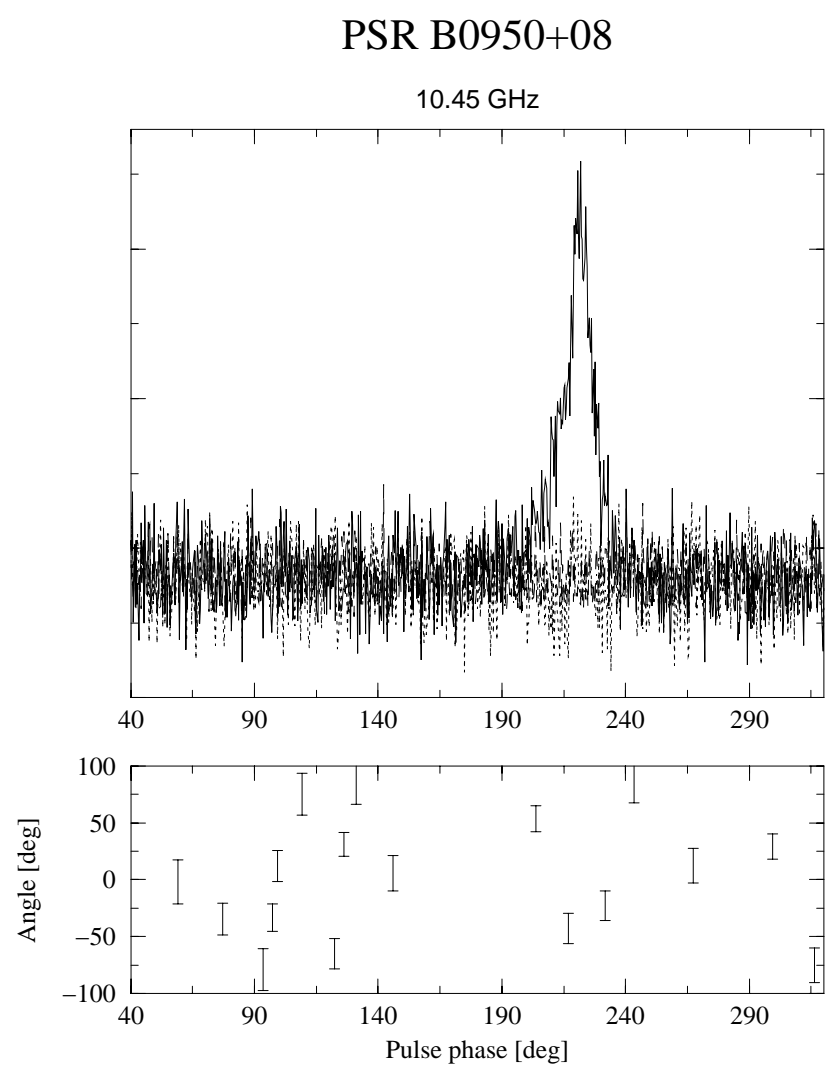

PSR B0950+08

$4.85 \mathrm{GHz}$
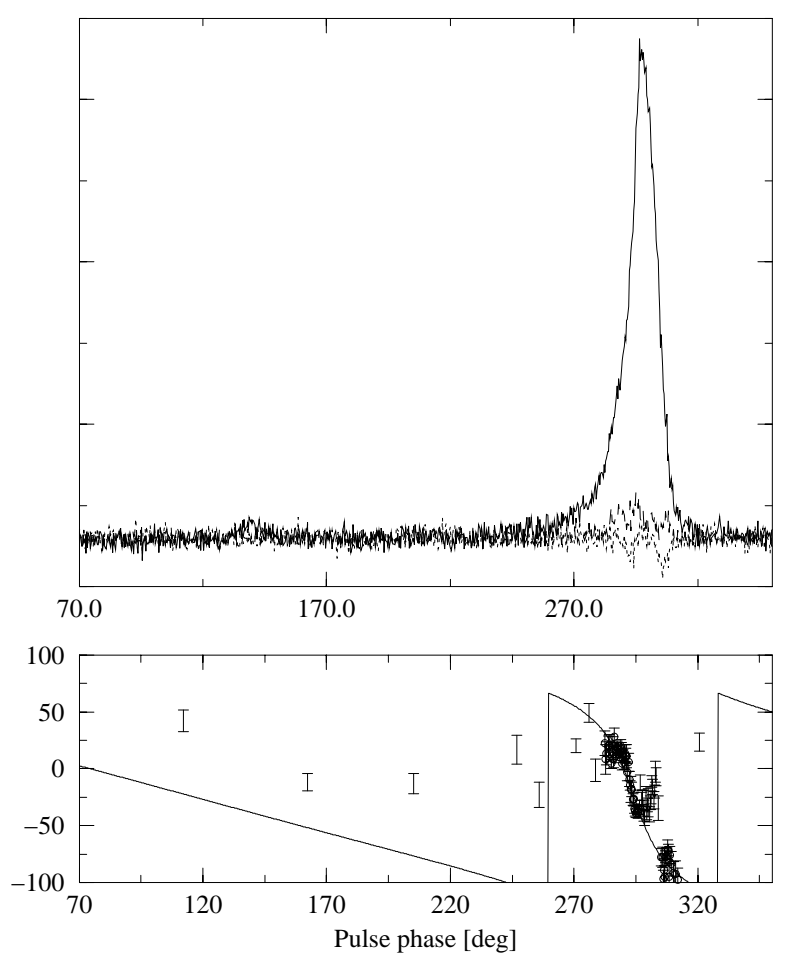

Fig. 16. PSR B0950+08 


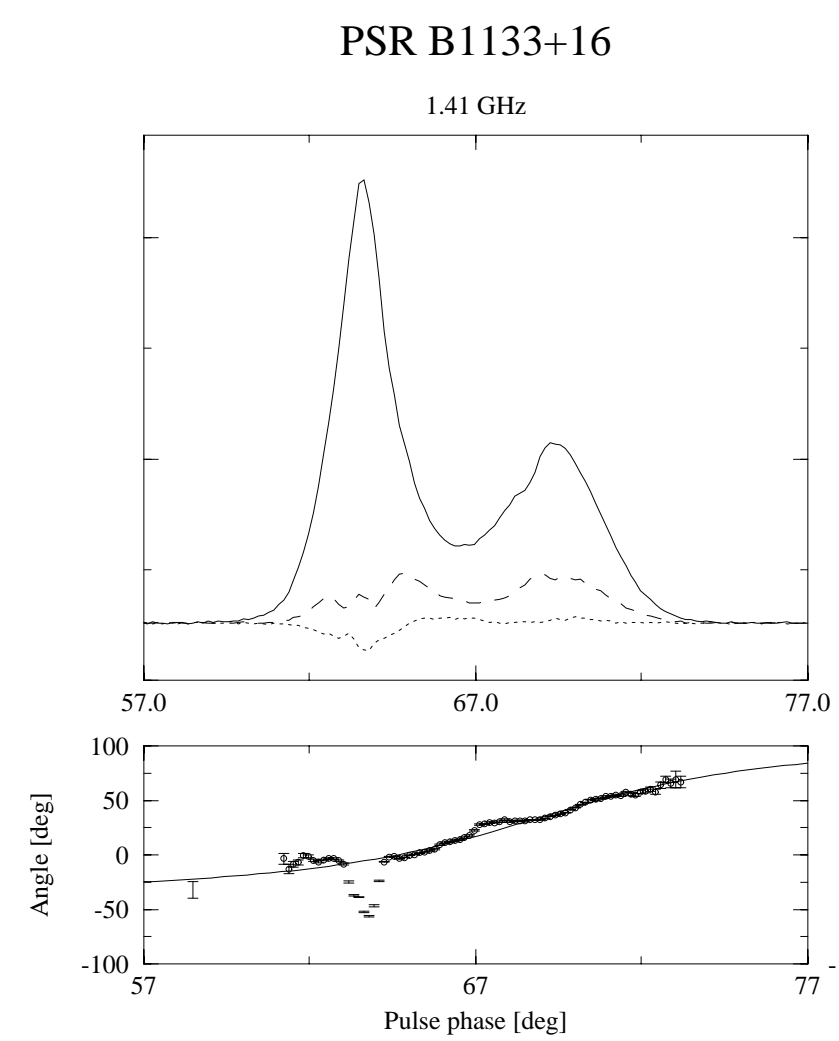

PSR B1133+16
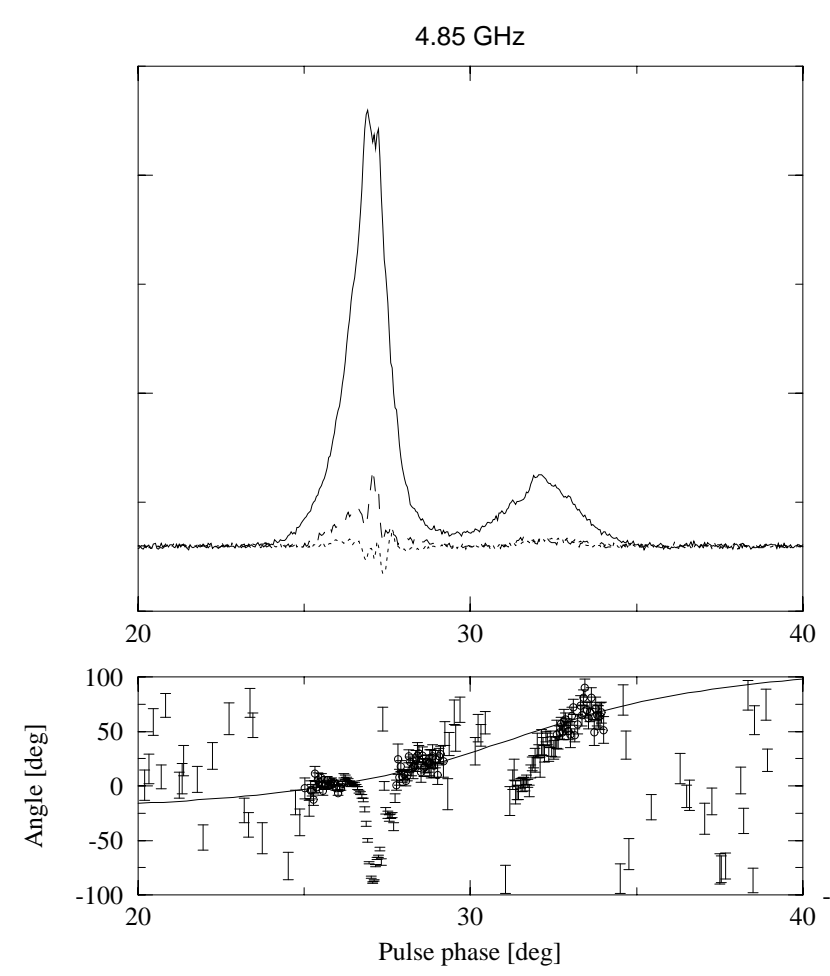

PSR B1133+16
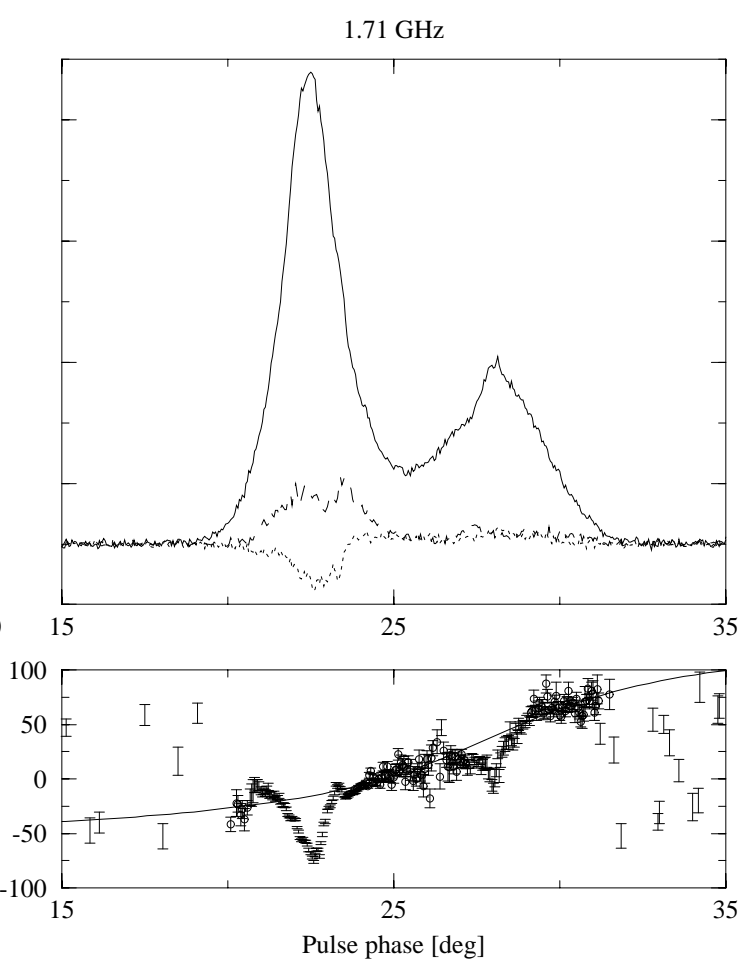

PSR B1133+16
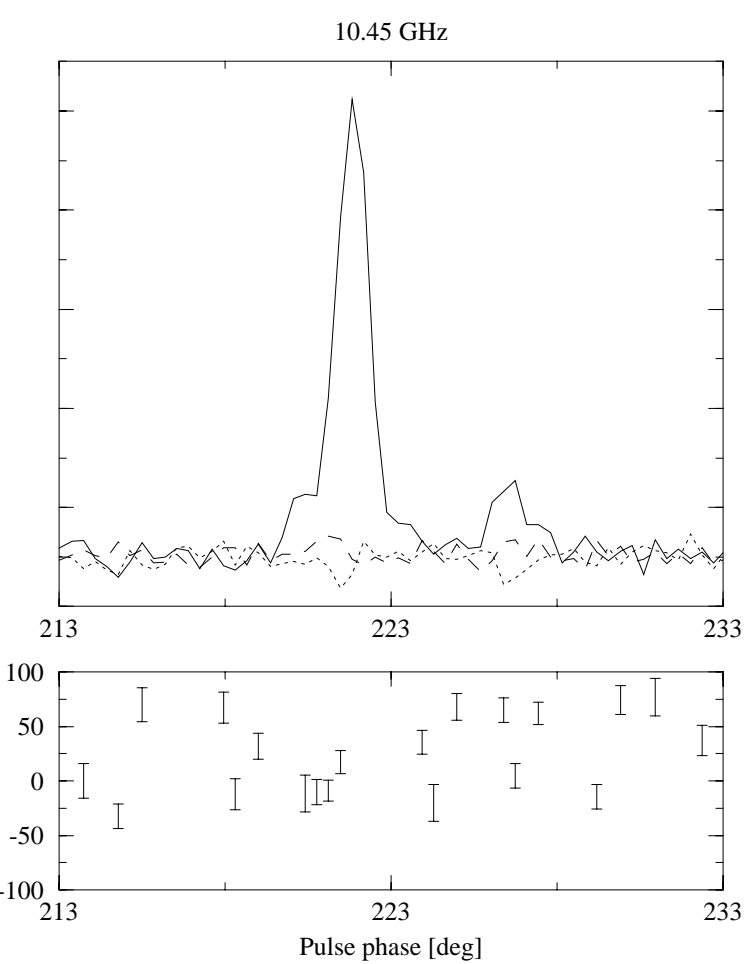

Fig. 17. PSR B $1133+16$ 
PSR B1822-09

$1.41 \mathrm{GHz}$
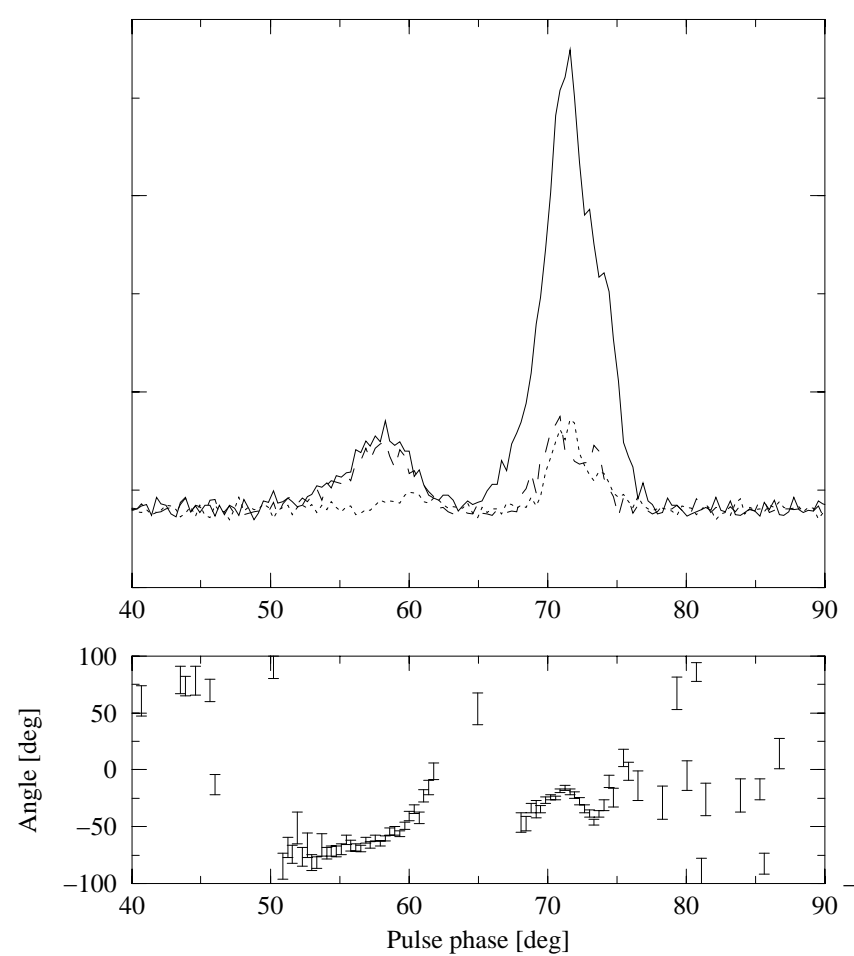

PSR B1822-09
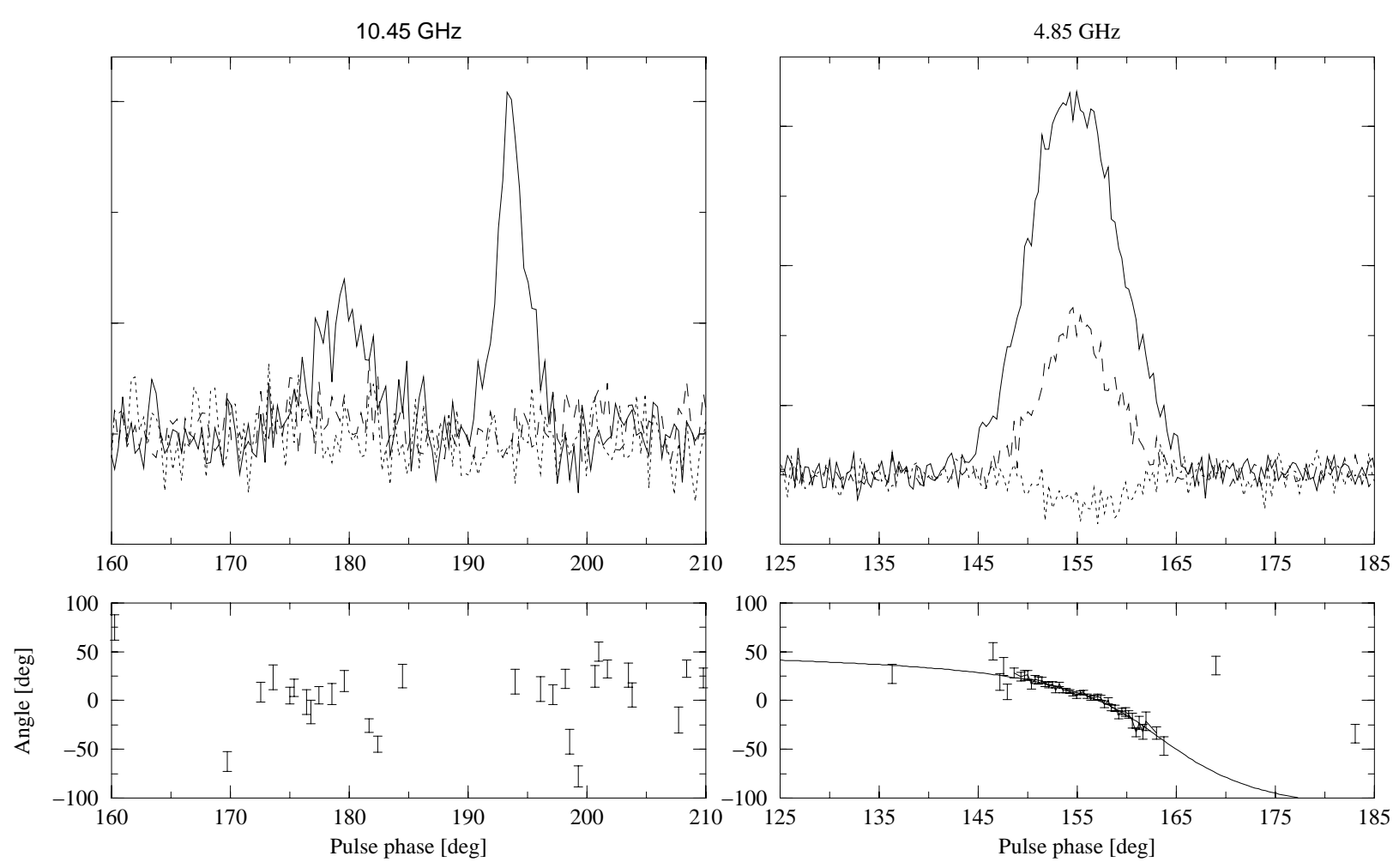

PSR B 1822-09
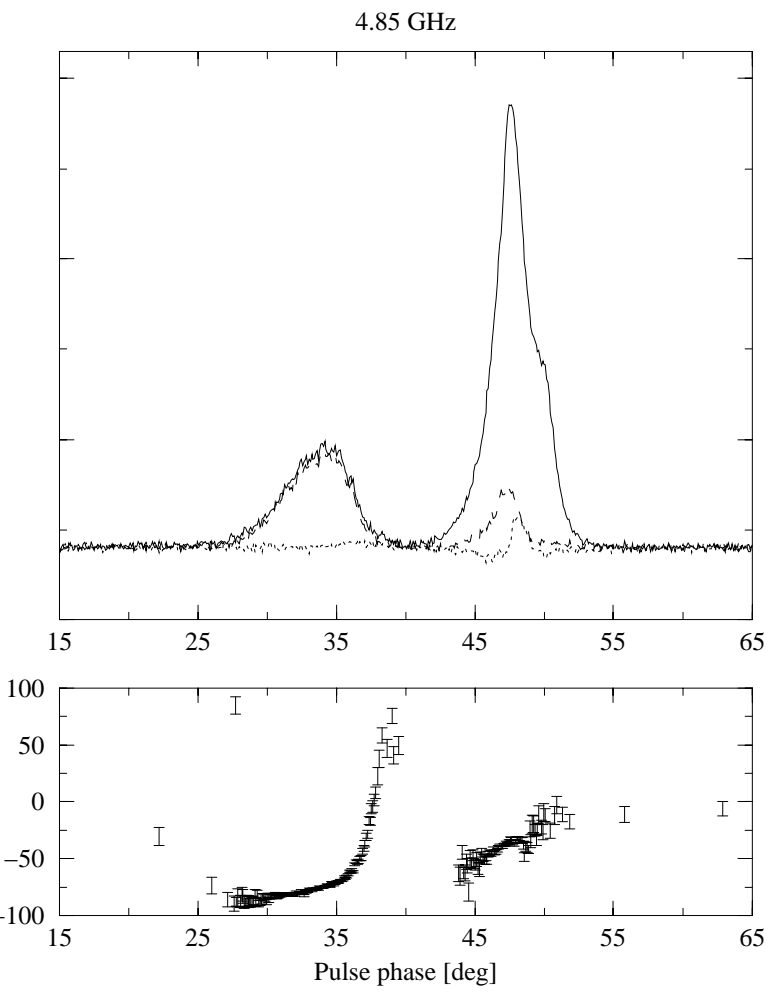

PSR B1915+13

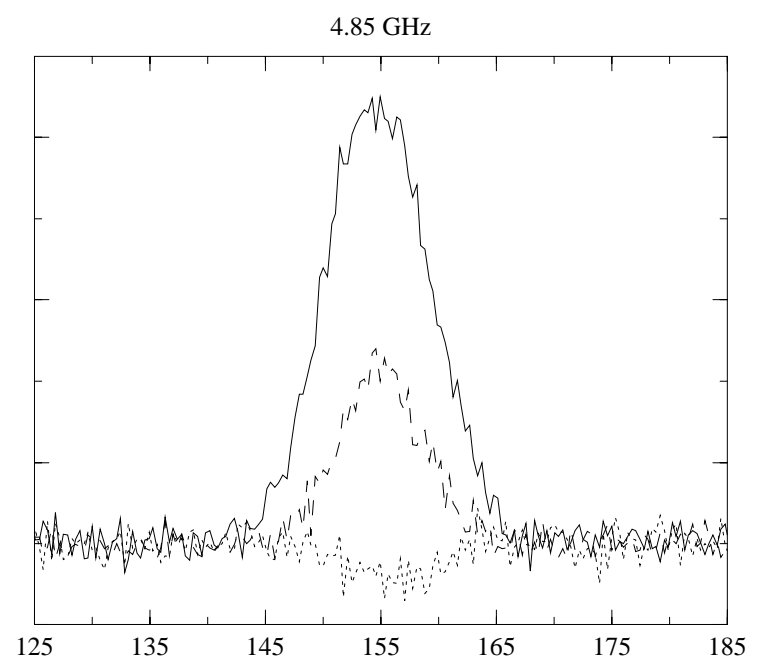

Fig. 19. PSR B1822-09 and PSR B1915+13 
PSR B1929+10

$1.41 \mathrm{GHz}$
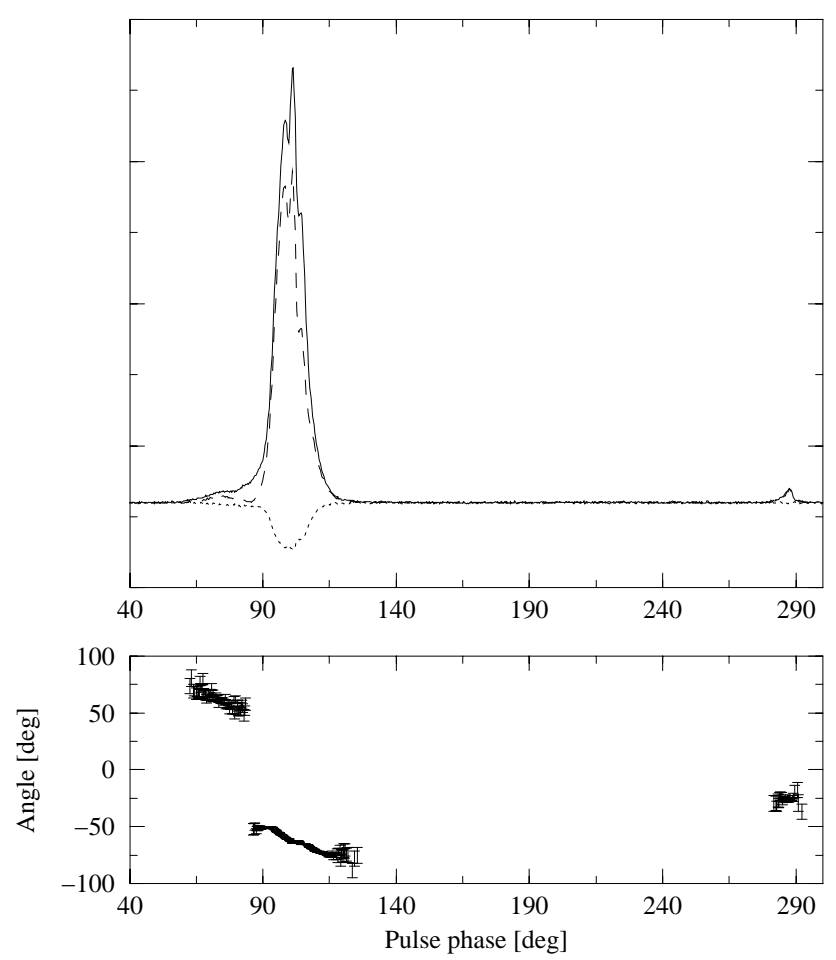

PSR B1929+10
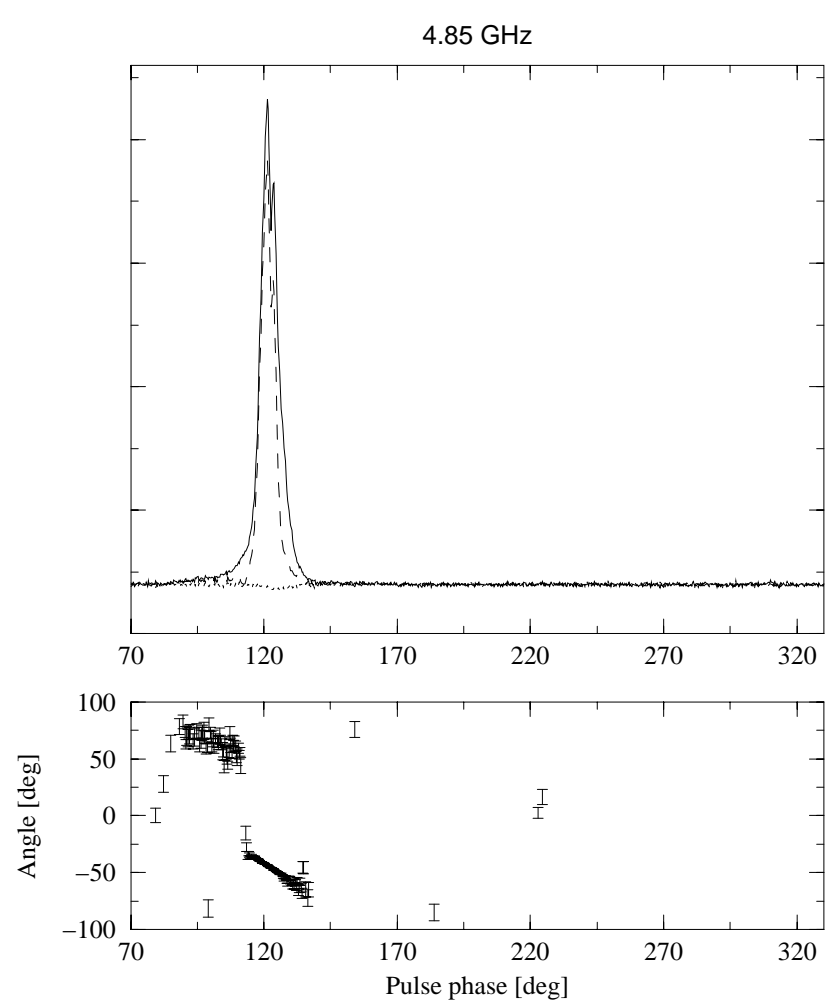

PSR B1929+10

$1.71 \mathrm{GHz}$
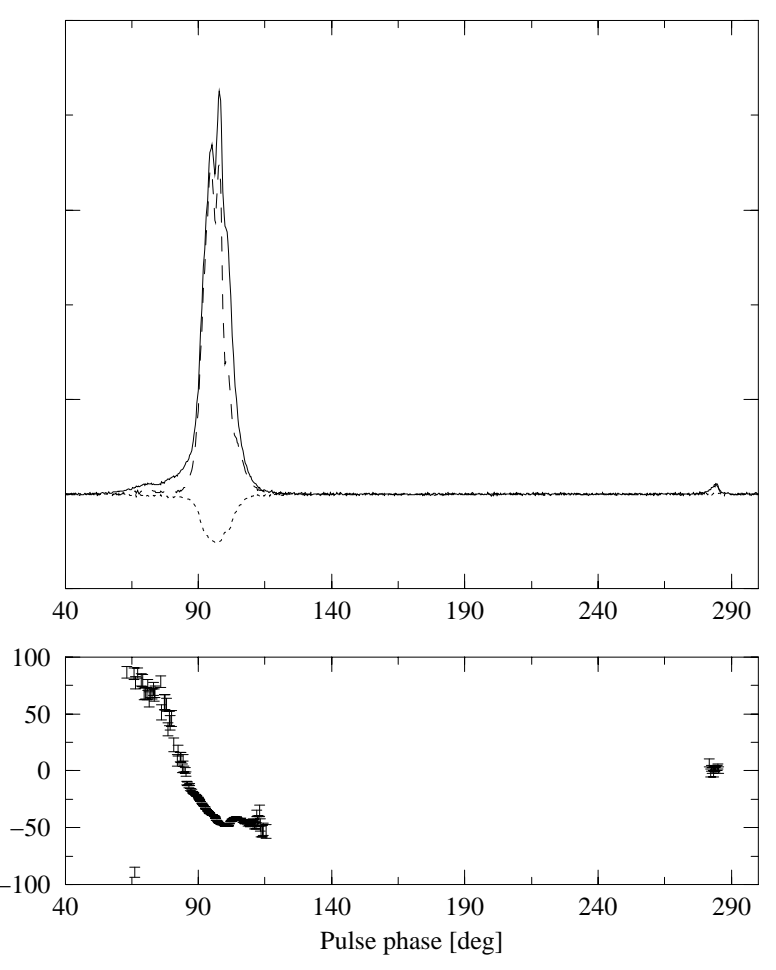

PSR B1929+10

$10.45 \mathrm{GHz}$
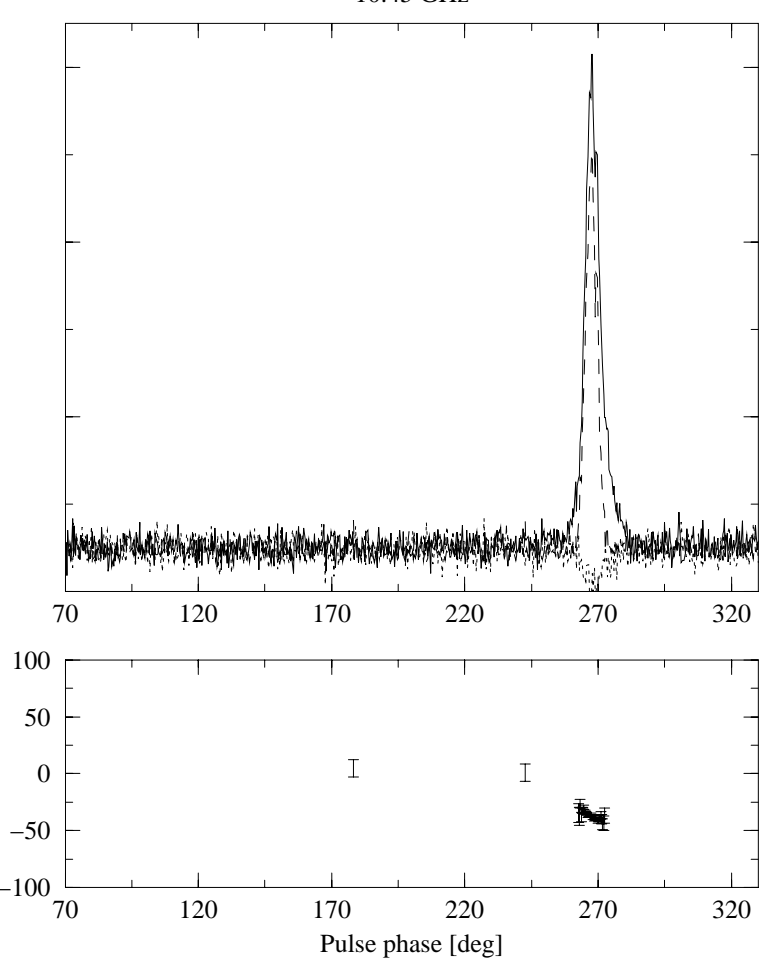

Fig. 20. PSR B1929+10 
PSR B1946+35

$4.85 \mathrm{GHz}$
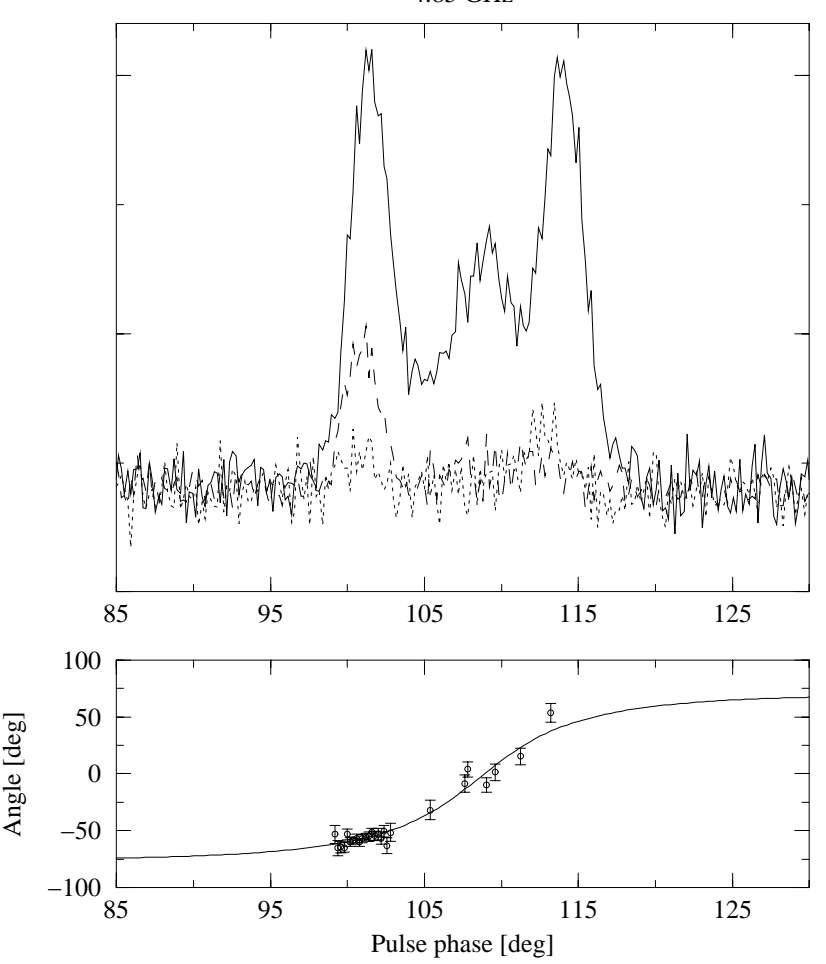

PSR B2020+28
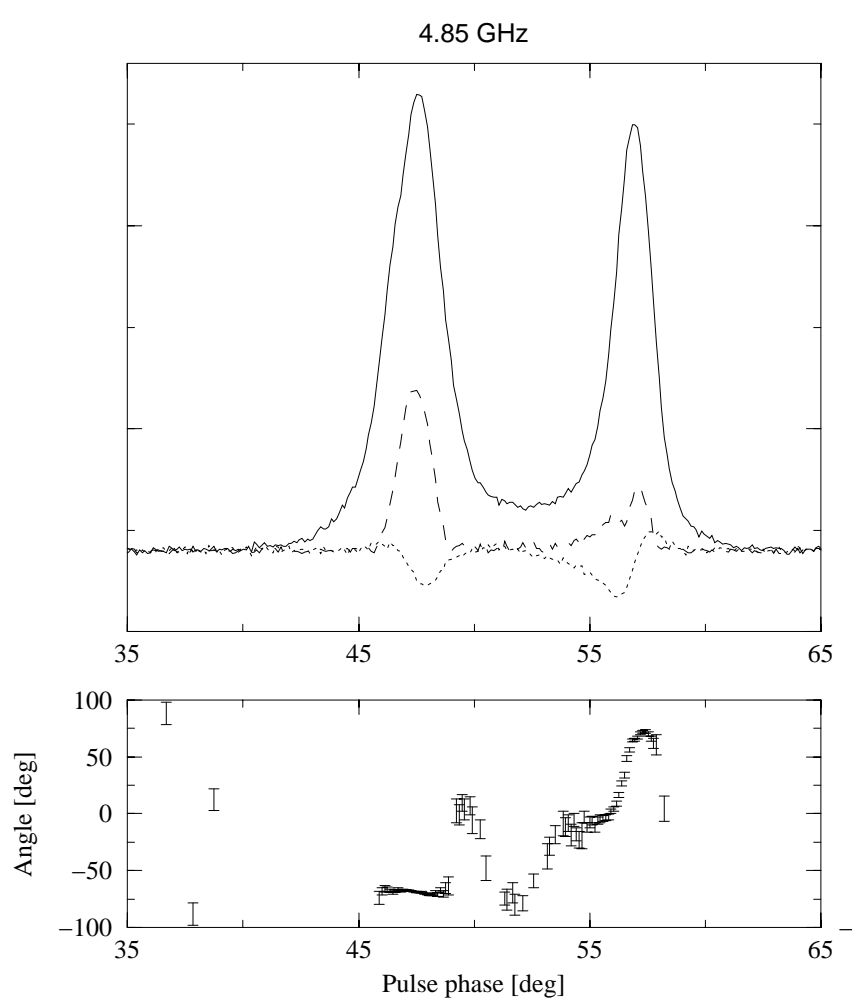

PSR B2016+28

$4.85 \mathrm{GHz}$
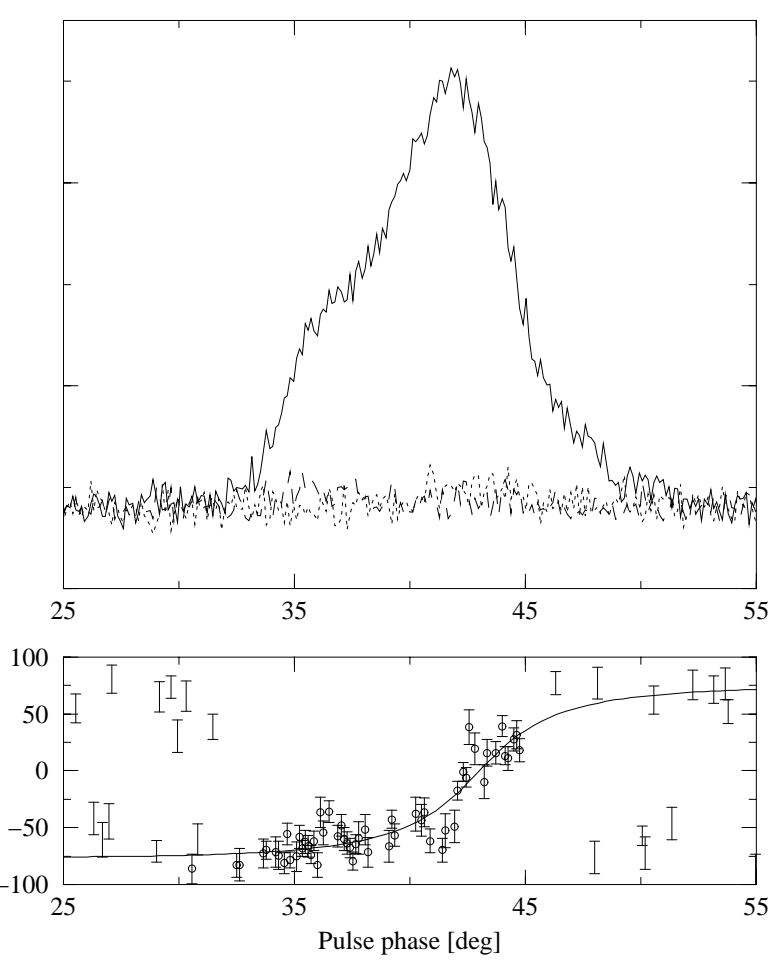

PSR B2020+28
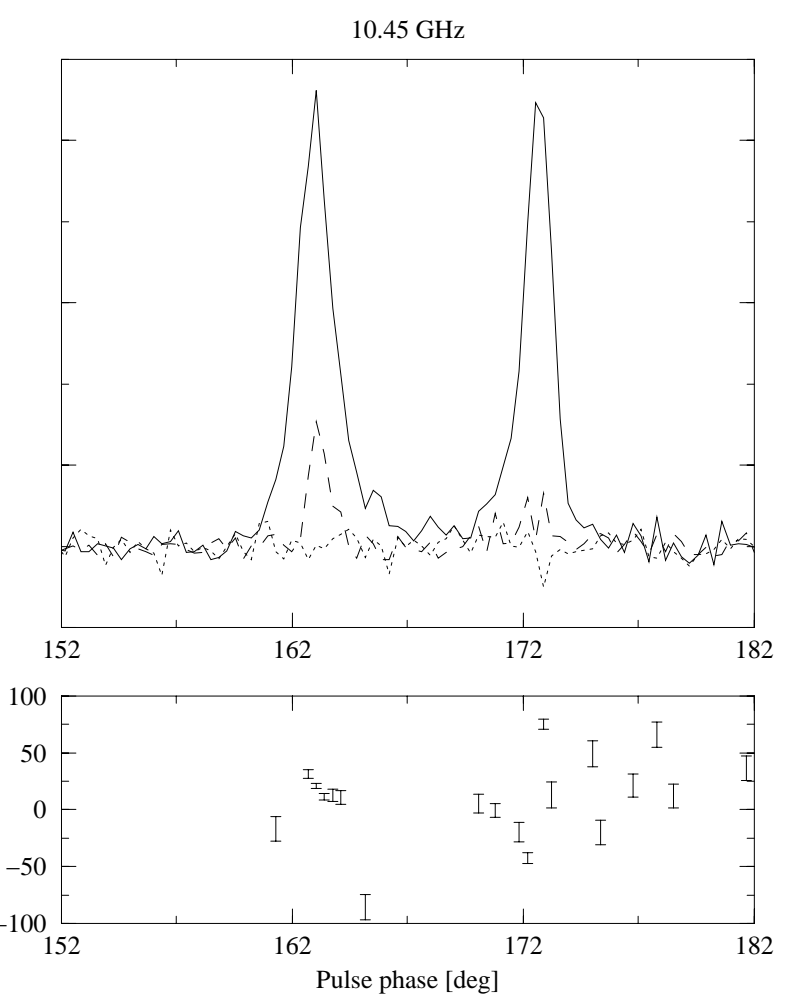

Fig. 21. PSR B1946+35, PSR B2016+28 and PSR B2020+28 
PSR B2021+51

$1.41 \mathrm{GHz}$
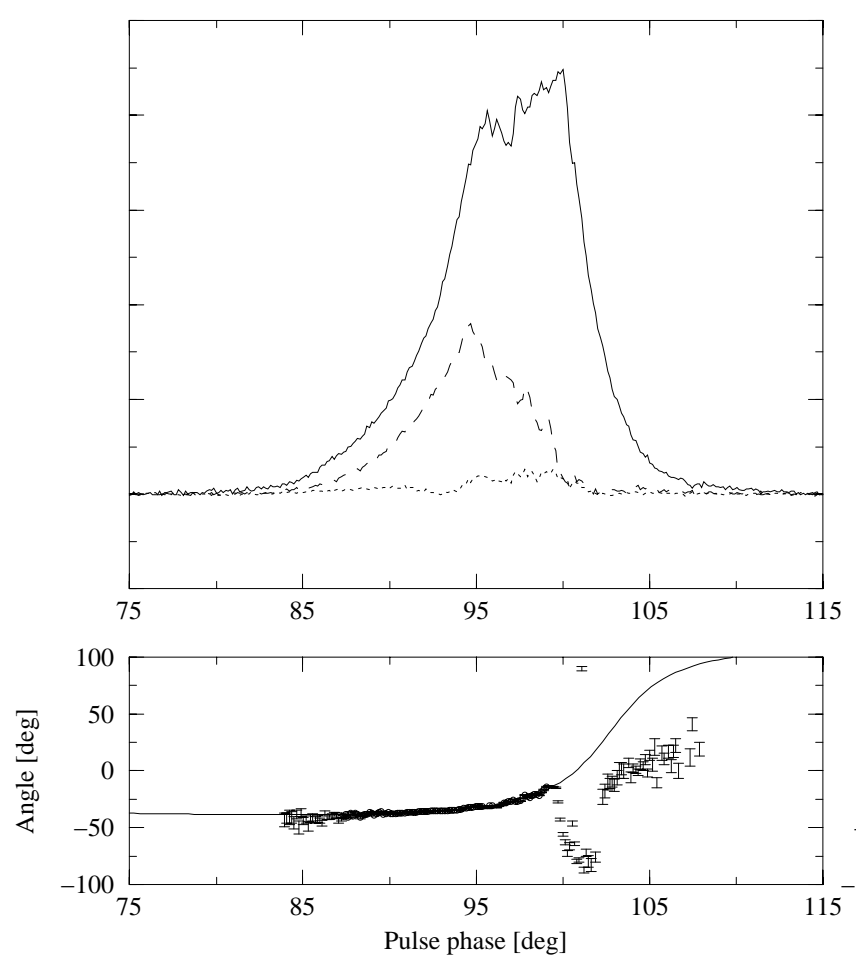

PSR B2021+51
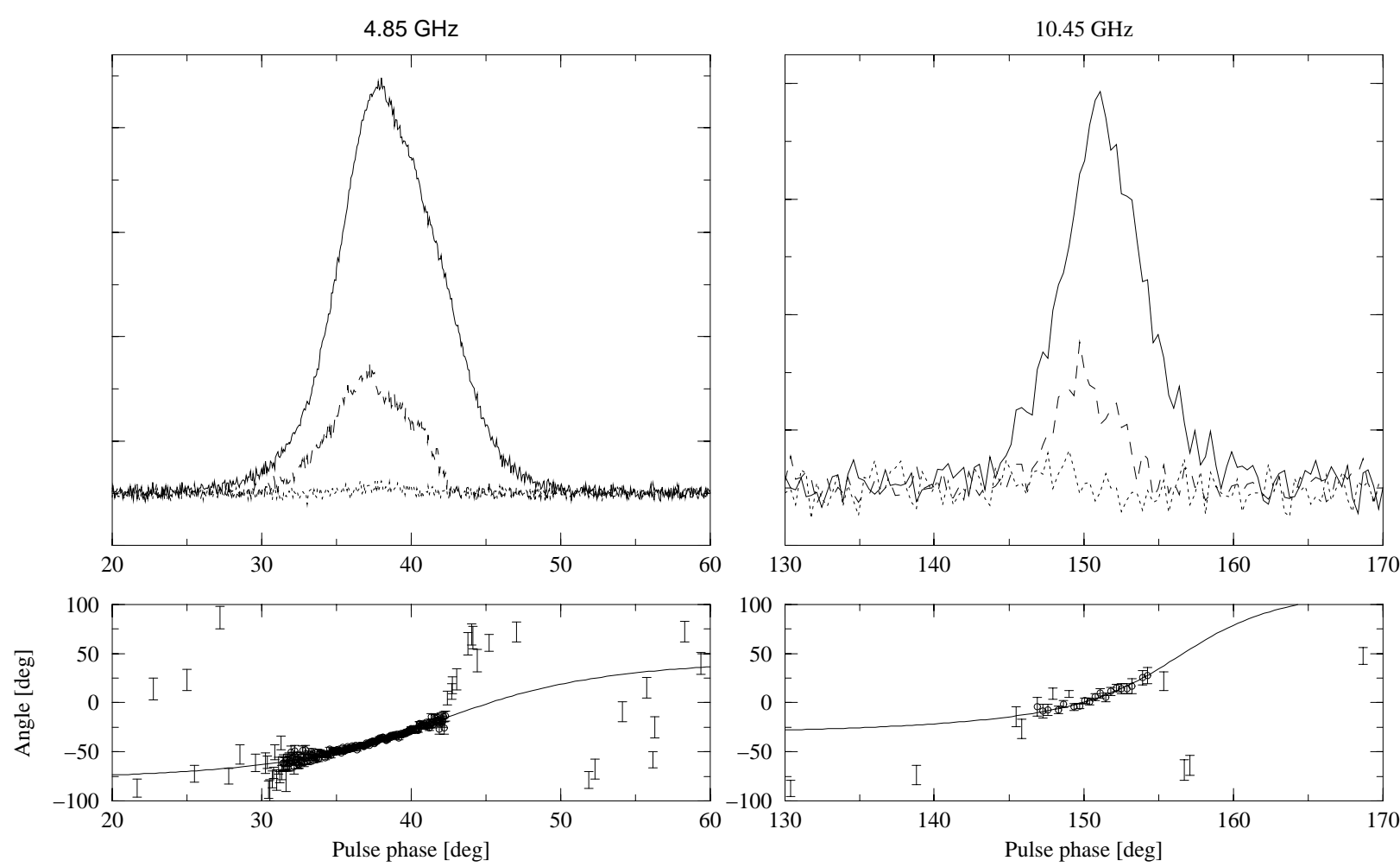

PSR B2021+51
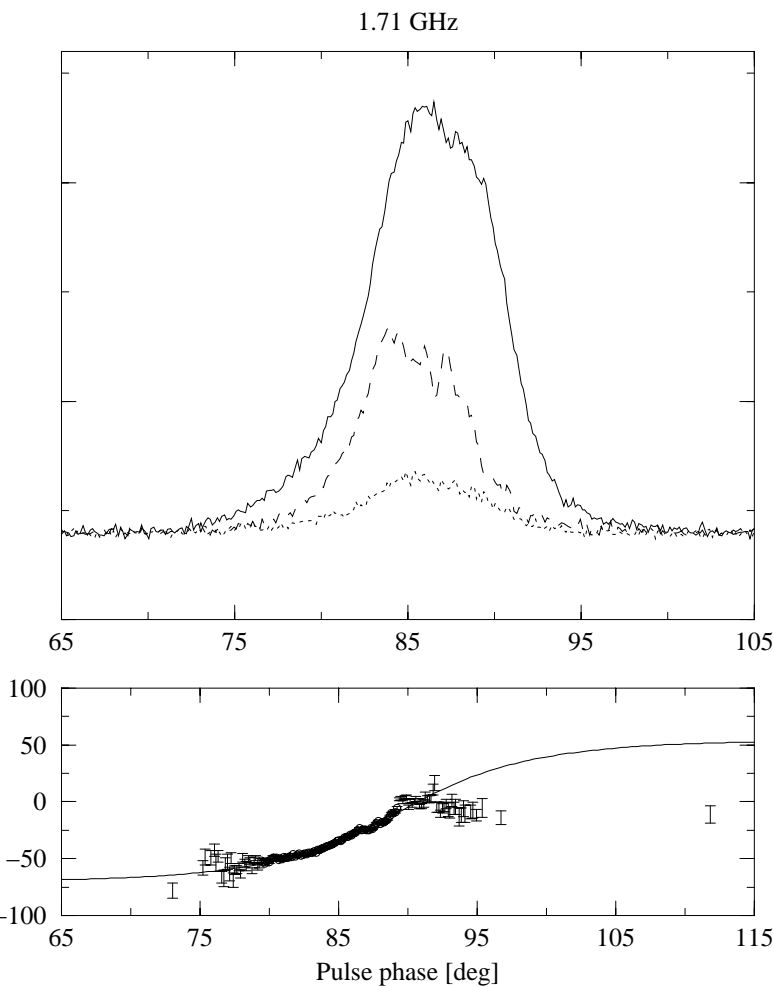

PSR B2021+51

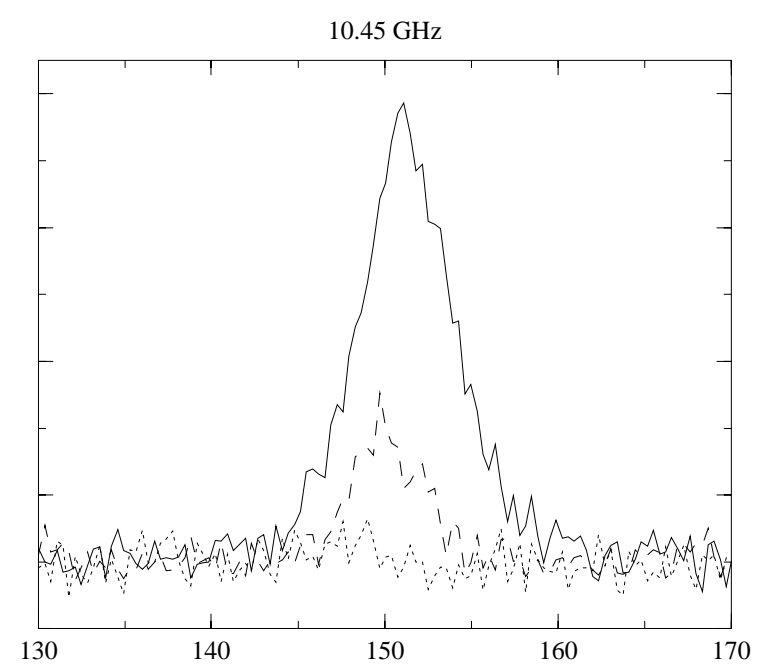

Fig. 22. PSR B2021+51

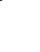


PSR B2045-16

$4.85 \mathrm{GHz}$
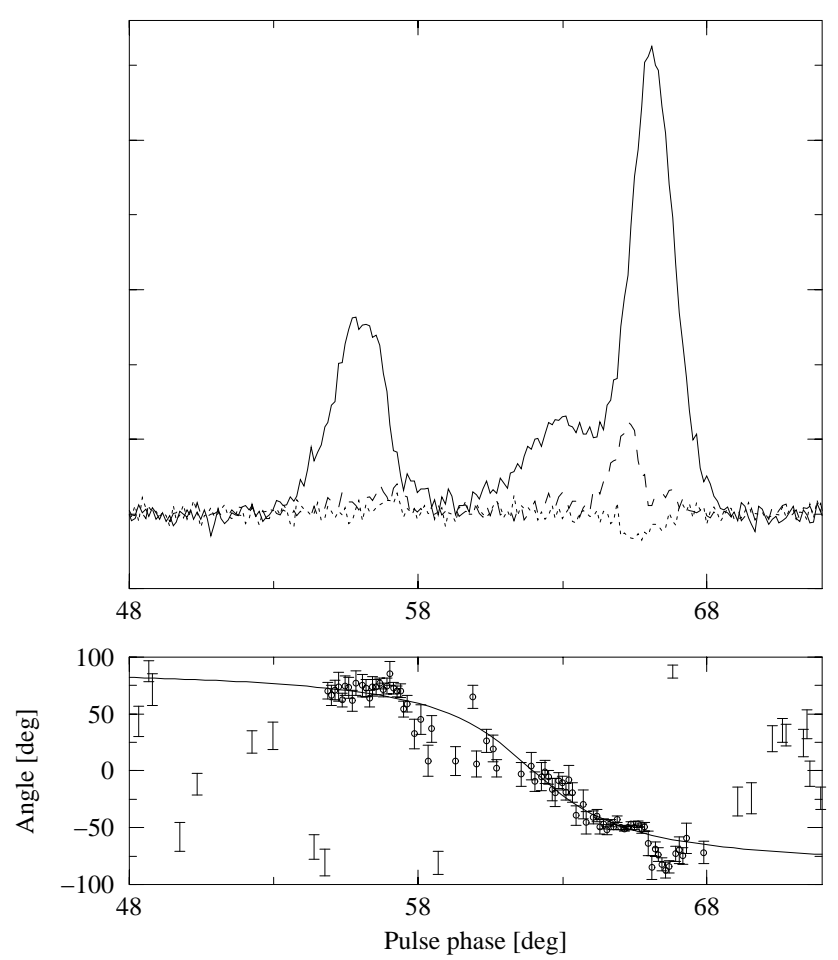

PSR B2310+42
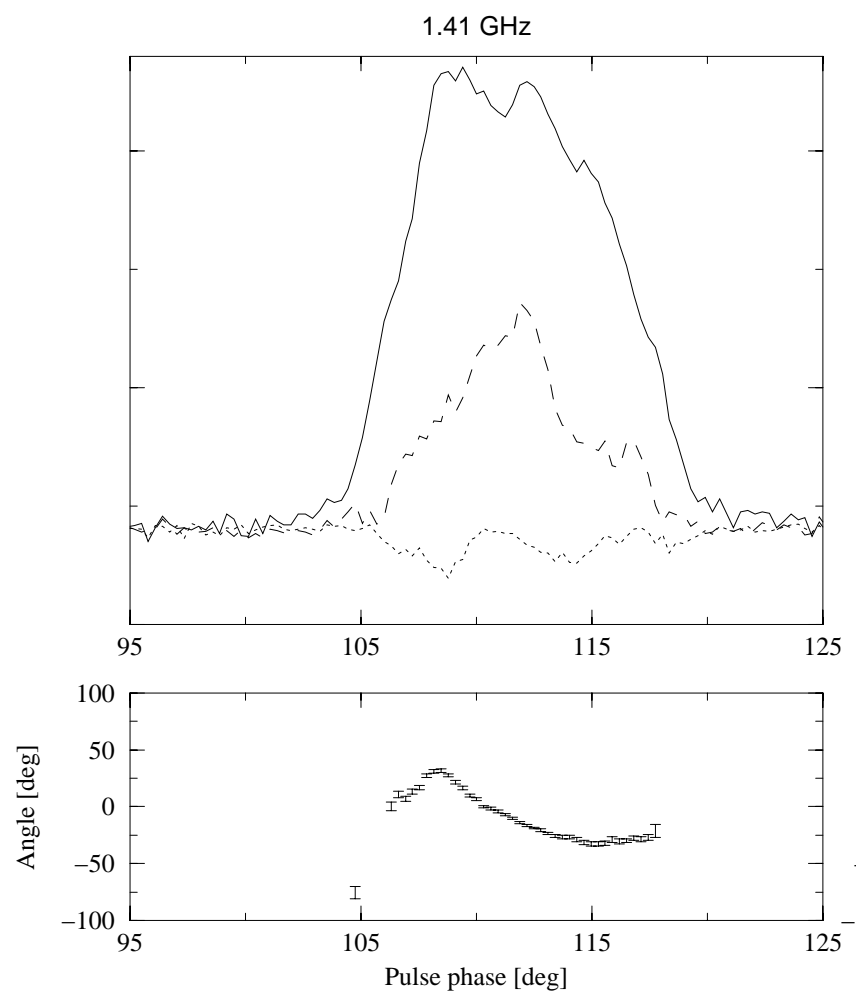

PSR B2154+40
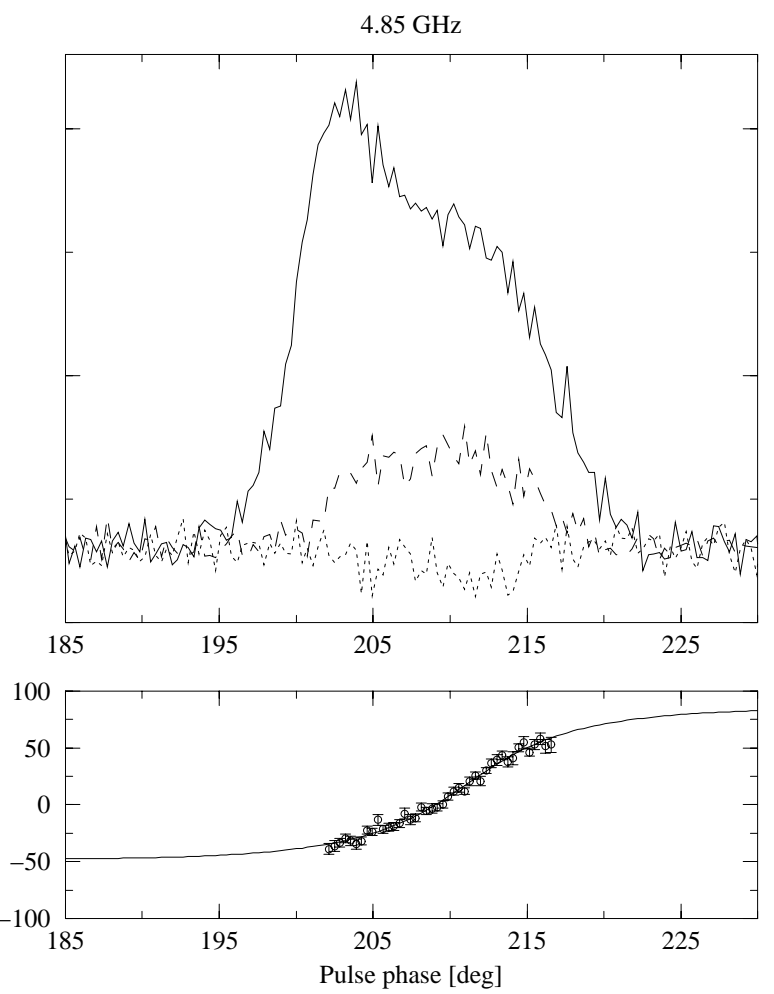

PSR B2310+42
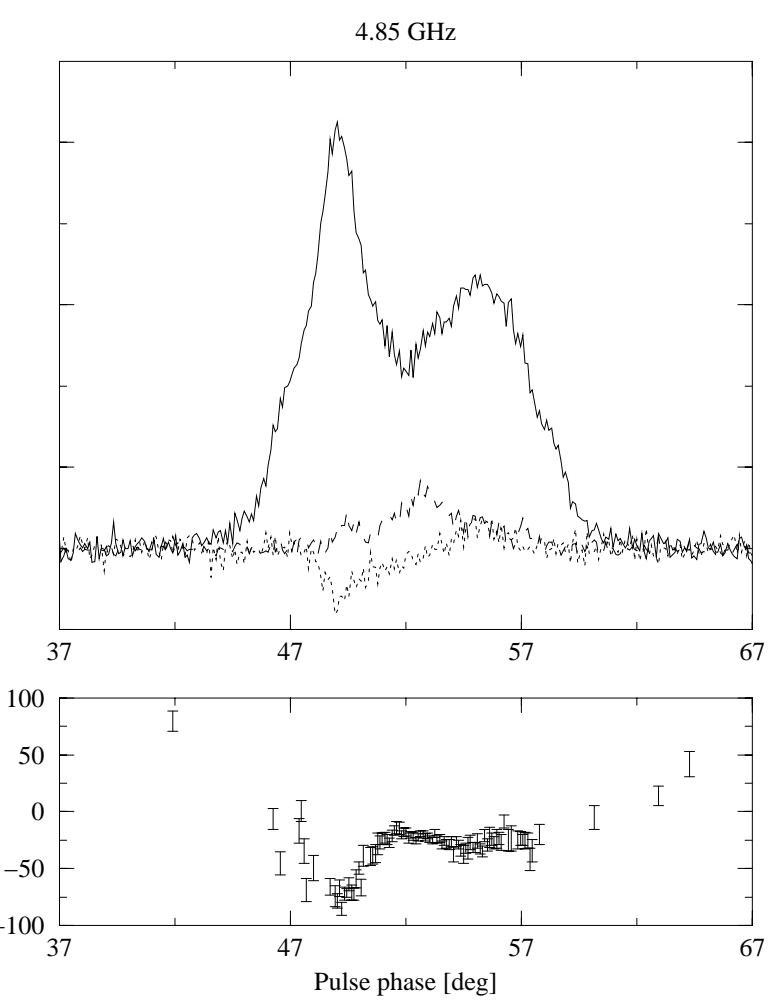

Fig. 23. PSR B2054-16, PSR B2154+40 and PSR B2310+42 
PSR B2319+60

$1.41 \mathrm{GHz}$
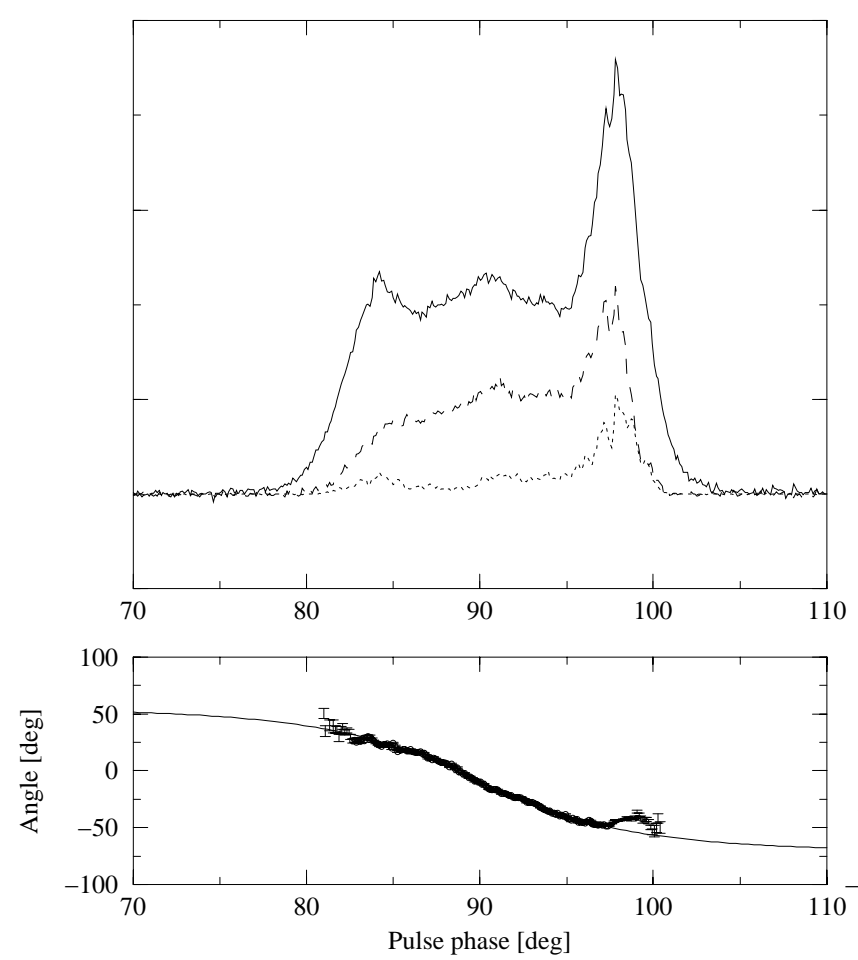

PSR B2351+61
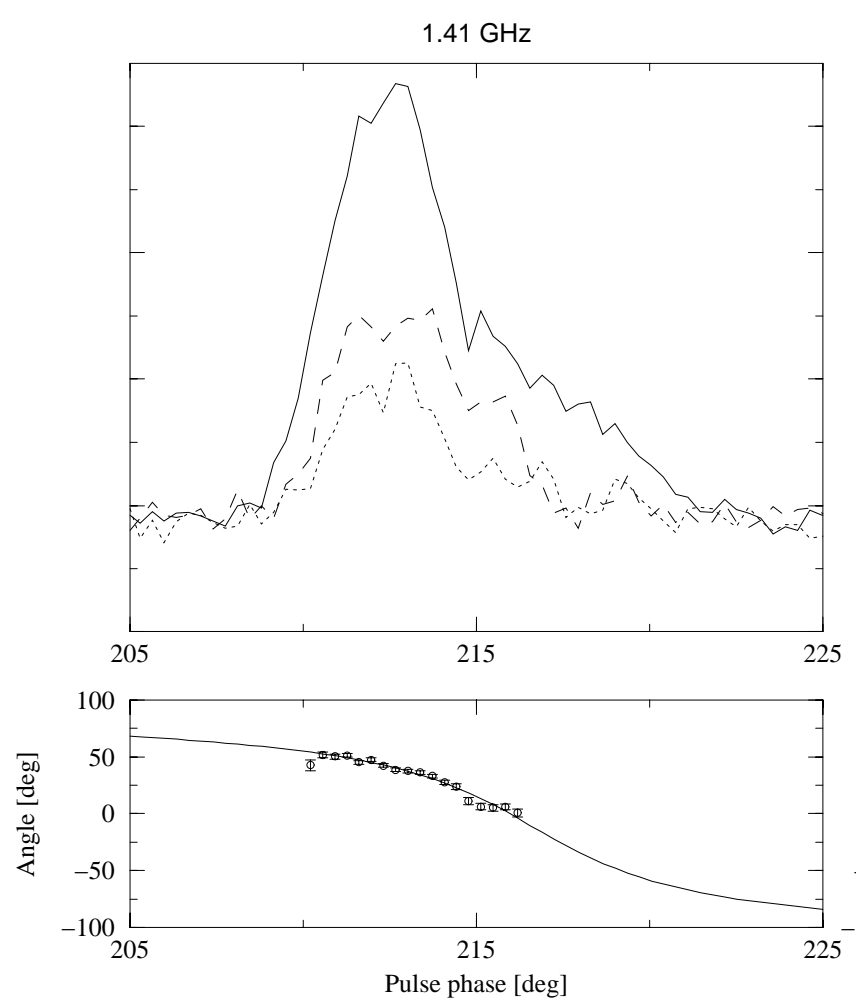

PSR B2319+60
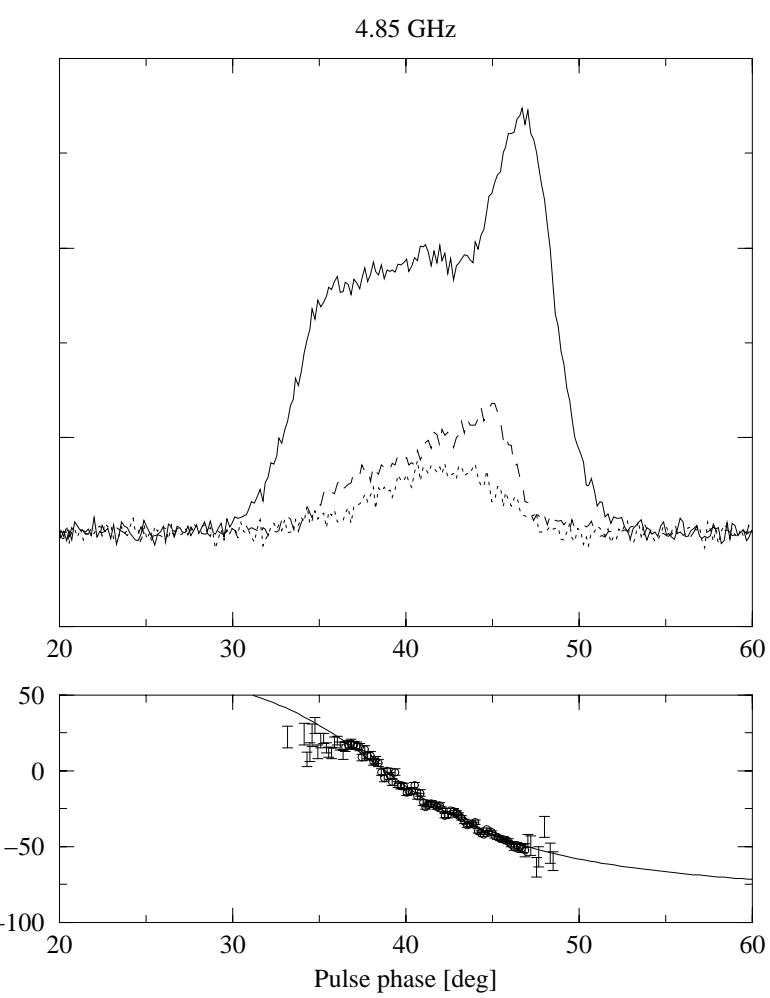

PSR B2351+61
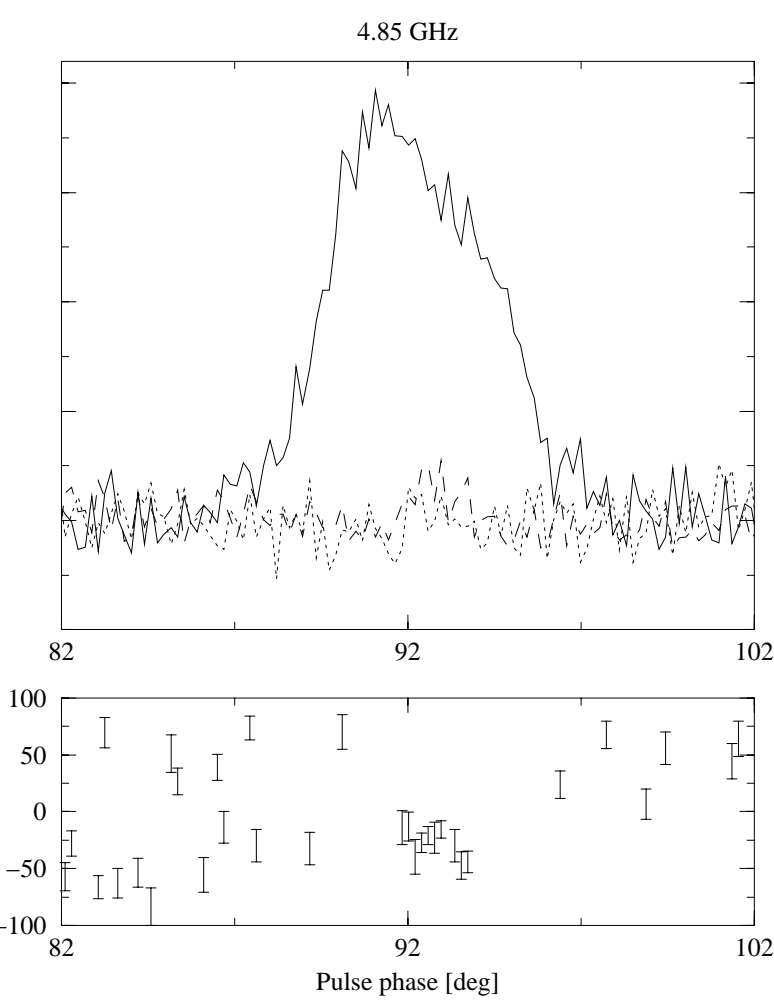

Fig. 24. PSR B2319+60 and PSR B2351+61 